\title{
Correlation between speed and turning naturally arises for sparsely sampled cell movements
}

\author{
Vitaly V. Ganusov ${ }^{1,2}$, Viktor S. Zenkov ${ }^{3}$, and Barun Majumder ${ }^{1}$ \\ ${ }^{1}$ Department of Microbiology, University of Tennessee, Knoxville, TN 37996, USA \\ ${ }^{2}$ Department of Mathematics, University of Tennessee, Knoxville, TN 37996, USA \\ ${ }^{3}$ Department of Electrical Engineering and Computer Science, University of Tennessee, Knoxville, TN \\ 37996, USA
}

May 8, 2021

\begin{abstract}
Mechanisms regulating cell movement are not fully understood. One feature of cell movement that determines how far cells displace from an initial position is persistence, the ability to traverse in a direction similar to previous direction, determined by turning angles between two sequential displacements. Recent studies found that a cell's average speed and turning are negatively correlated, suggesting a fundamental cell-intrinsic program whereby cells with lower turning ability (i.e., larger persistence) are intrinsically faster. Using simulations, we show that a negative correlation between the measured average cell speed and turning angle naturally arises for cells undergoing a correlated random walk due to sub-sampling, i.e., when frequency of sampling is lower than frequency at which cells make movements. Assuming heterogeneity in persistence and intrinsic speeds of individual cells results in a negative correlation between average speed and turning angle that resembles experimentally observed correlations. Changing the frequency of imaging or calculating displacement of cohorts of cells with different speeds resulted in similar results whether or not there is a cell-intrinsic correlation between cell speed and persistence, and we find many different parameter sets that allow to approximately match experimental data binned into cell cohorts. Interestingly, reanalysis of $\mathrm{T}$ cell movements in zebrafish showed that the observed correlation between persistence and speed is sensitive to sampling frequency, disappearing for coarsely sampled data. Our results challenge an established paradigm that persistent cells have intrinsically faster speeds and emphasize the role of sampling frequency have on inference of critical cellular mechanisms of cell motility.
\end{abstract}

Significance Statement: Measurement of cell movements often results in a negative correlation between average speed and average turning angle suggesting an existence of a universal, cell-intrinsic movement program. We show that such a negative correlation may arise if cells in the population differ in their ability for persistent movement when the movement data are sub-sampled. We show that such a model is consistent with the published experimental data and provide potential ways of how the sub-sampling model can be falsified.

Abbreviations: MSD - mean squared displacement; VMF - von Mises-Fisher, TA - turning angle. 


\section{Introduction}

Motility is a fundamental property of cells. Motility is exhibited by single cell organisms such as bacteria or ameba as well as by cells of multicellular organisms such as cells during development or cancer [1-4]. To protect the host against pathogens $\mathrm{T}$ lymphocytes, cells of the adaptive immune system need to constantly move and survey tissues for infection $[5,6]$. While molecular mechanisms of $\mathrm{T}$ cell motility have been relatively well defined, specific strategies that $\mathrm{T}$ cells use to efficiently locate the pathogen remain controversial. Some studies indicated that $\mathrm{T}$ cells search for the infection in the lymph nodes randomly [7,8] while others suggested an important role of attracting cues such as chemokines $[6,9]$. In non-lymphoid tissues, a wider range of movement types of $\mathrm{T}$ cells have been observed, including Brownian walks in the skin [10], correlated random walks in the liver [11] or in explanted lungs [12], or generalized Levy walks in murine brains [13].

Movement of cells in tissues in vivo is typically recorded using intravital microscopy at a particular frame rate (e.g., a small 3D volume of the liver or a lymph node of $500 \times 500 \times 50 \mu \mathrm{m}$ can be scanned with a two photon microscope every $20 \mathrm{sec},[11,14,15])$. By segmenting images with software packages (e.g., ImageJ from the NIH or Imaris from Bitplane), 3D coordinates of multiple cells over time can be obtained. Generation of 3D cell coordinates from raw imaging data is a complicated process, and in some cases z-coordinates are either ignored or collapsed into one using maximal projection, thus resulting only in change in $x$ and $y$ coordinates for individual cells over time [16-18]. Cell coordinates can be then used to characterize movement pattern of the cells. Several alternative parameters are useful in this regard $[17,19]$. One is the distribution of movement lengths, which when is normalized by the imaging frequency, gives the distribution of instantaneous speeds of cells from which the average speed per cell can be calculated. Distribution of movement lengths can be used to infer heterogeneity in cell migration speeds or indicate if cells are utilizing a specific search strategy such as Levy walks $[11,13,16,20,21]$. It is important to note, however, that estimated average or instantaneous speeds of cells are generally dependent on the frequency at which imaging is performed $[22]$.

Another important parameter characterizing cell movement is persistence - the ability of cells to keep moving in the direction similar to the previous movement direction; such a walk pattern is also defined as a correlated or persistent random walk. While intuitively cell persistence is a relatively clear concept, ways to characterize how "persistent" cells in a given population are, vary. One approach is to perform linear regression analysis on the initial change in the log-transformed mean square displacement (MSD) of cells vs. log-transformed time and find the time $P$ until which the slope in this initial change is larger than 1 [18]. A complementary approach is to calculate the slope in the exponential decline of the correlation between cell's velocity vectors over time when averaged over all cells in the population; an inverse value of the slope also gives the average persistence time $[17,18]$. An alternative approach to characterize the ability of cells in the population to undergo a correlated random walk is calculate turning angles - angles that a cell takes when making sequential movements as recorded in an experimental movie [19, 22, Figure 1]. The distribution of turning angles along with the movement length distribution thus characterizes the movement pattern of cells in the population. The average turning angle $\bar{\phi}$ may then inform the ability of cells in the population to persistently move: when the average turning angle is $\bar{\phi}=90^{\circ}=\pi / 2$ (and the turning angle distribution is described by a sin function), cells are not persistent and perform a Brownian random walk. When $\bar{\phi}<90^{\circ}$, cells perform a correlated random walk. The fraction of cell movements with turning angles higher than $90^{\circ}$ also indicates the probability of cells to turn away from the previous 
movement direction. However, as compared to the estimated persistent time $P$, the average turning angle $\bar{\phi}$ does not represent an intuitive parameter indicating how long cells are persistent in their movement.

What determines the ability of cells to exhibit correlated random walks remains poorly understood. We recently argued that the constrained environment of the liver sinusoids is likely to force liver-localized CD8 T cells to perform correlated random walks resulting in super-diffusive behavior for tens of minutes [11]. However, the ability of cells to perform a correlated random walk could be cell-intrinsic. Indeed, authors of several recent studies accurately measured movement of cells in 2D (in vitro) or in 3D (in vivo) over time and found that there is a strong positive correlation between average cell persistence, defined as the persistence time or the cosine of turning angles, and average cell speed [18, 23, 24]; this is equivalent to the negative correlation between average turning angle per cell and cell speed. Specifically, experiments by Jerison \& Quake [18] were designed to monitor the migration of $\mathrm{T}$ cells in vivo in zebrafish for hours given the transparency of the animal and methods to stitch different images across nearly the whole animal. Their analysis of the data suggested that $\mathrm{T}$ cells exhibited large heterogeneity in movement speeds, cohorts of cells with similar speeds showed different persistence times, and there was a strong negative correlation between average turning angle per cell and average speed [18, see also Results section], suggesting a fundamental property of cell movement: cells that turn less have intrinsically larger speeds [18, 23]. Importantly, the correlation between turning ability and speed was observed for different cell types, including unicellular organisms, and genetic perturbations or treatment of cells with drugs impacting cell movement ability did not eliminate the observed correlation, suggesting that indeed the relationship between persistence and speed are fundamental to cell movement $[18,23]$.

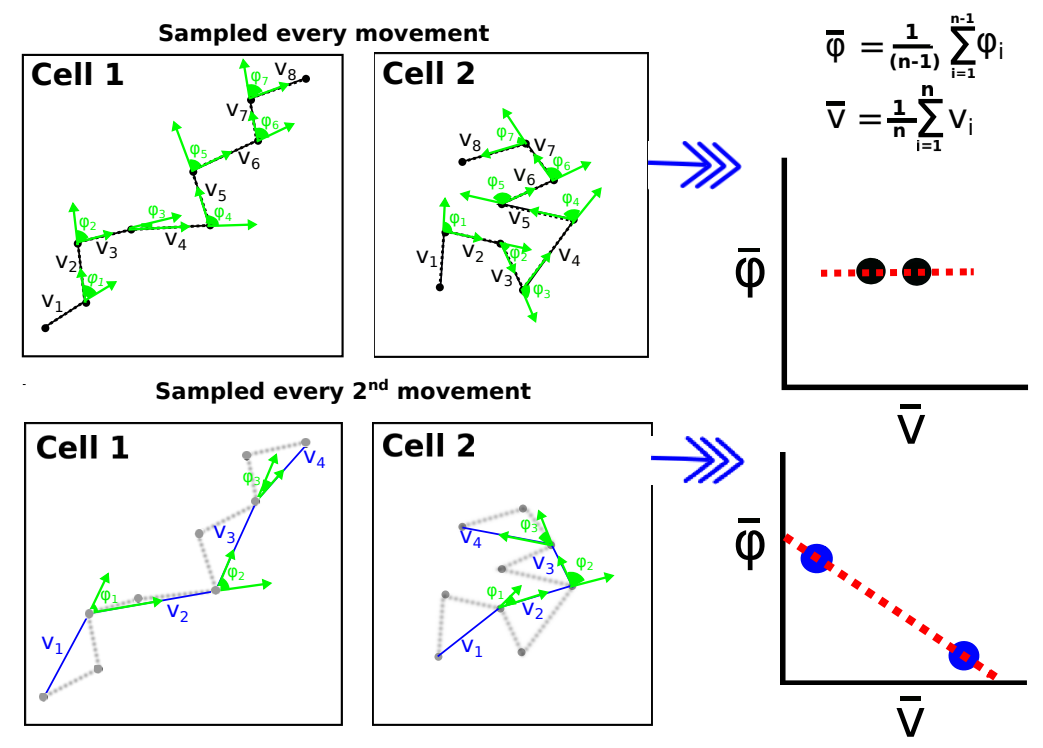

Figure 1: Schematic representation of how frequency of sampling of cell movement influences the observed relationship between average speed and average turning angle per cell. We plot trajectories for 2 cells and show the speed of every movement (denoted as $v_{i}$ ) and turning angle $\left(\phi_{i}\right)$. When sampling is done at the same frequency as cells make a decision to turn (top panels), per model assumption average speed $(\bar{v})$ and average turning angle $(\bar{\phi})$ do not correlate. However, when sampling is done less frequently (e.g., every 2nd movement, bottom panels) the cell 1 that turned less has a lower average turning angle and higher observed speed compared to the cell 2 , which turned more.

It is important to remember that both cell persistence (evaluated, say, by average turning angle) and cell speed are estimated parameters from experimental data, and the true turning ability and 
true instantaneous speeds of cells are not generally known. The frequency at which individual cells make decisions to change direction of movement and movement speed is also unknown, especially given imprecise measurements of cell positions at a high frequency of imaging. Here we shown that the experimentally observed negative correlation between average turning angle and average speed per cell may arise due to coarse sampling of cell movement trajectories when cells undergo a correlated random walk (Figure 1). Indeed, when cells undergo a correlated random walk some cells may displace far and some will remain confined due to random chance alone, and when trajectories are sub-sampled, one expects to see a negative correlation between average turning angle and average speed (Figure 1). Furthermore, we show that when there is a variability in persistence ability between individual cells (but not a variability in speed), the negative correlation between average turning angle and average speed is observed for a large range of speeds, which is consistent with experimental observations. Importantly, none of the conventionally used parameters such as MSD for cell cohorts or the slope between average turning angle and average speed allowed us to discriminate between the two models in which there is or there is not a cell-intrinsic link between persistence and speed. We also show that the negative correlation between average turning angle and speed strongly depends on the imaging frequency and disappears for sub-sampled experimental data on the movement of $\mathrm{T}$ cells in zebrafish. Our results thus challenge the established paradigm that persistent cells have intrinsically faster speeds.

\section{Results}

Correlation between persistence and speed arises naturally in simulations with subsampled data. Because cells' intrinsic programs for speed and ability to turn are not known, a cell's speed and average turning angle are estimated using measurements. Geometrically, it is clear that if the sampling of a cell's positions occurs rarer than the times of decisions the cell makes to turn, a negative correlation between the estimated speed and estimated turning angle may naturally arise because cells that turned less by chance are likely to be observed to displace further, and thus have a higher estimated speed (Figure 1). To check this intuition, we ran a series of stochastic simulations.

First, we simulated 500 cells moving randomly via Brownian motion with movement lengths following a thin-tailed distribution (Pareto distribution with $\alpha=5.5$ and $\bar{r}=2$, [11, see Materials and Methods]). When the cells' positions were sampled at the frequency that the cells were changing movement direction, we observed no correlation between average speed and average turning angle as assumed (Figure 2A). Interestingly, with sampling every $10^{\text {th }}$ movement, we still observed no correlation between average speed and turning angle was observed (Figure 2B). The average speed of cells in such rarely sampled data was also about 4 times smaller than the assumed speed, because cells were turning and thus not displacing far from the initial location.

Second, to simulate a correlated random walk we used our newly proposed methodology of sampling random vectors with a bias towards a particular direction using the von Mises-Fisher (VMF) distribution [25, see Materials and Methods]. When we allowed for a moderately biased correlated random walk (with concentration parameter $\kappa_{t}=1$ in VMF distribution), we did not observe a statistically significant correlation between average turning angle and average speed in sub-sampled data (results not shown). However, for a more biased correlated random walk $\left(\kappa_{t}=10\right)$, we observed that rarer but not regular sampling of cells' positions resulted in a statistically significant but weak negative correlation between measured speed and the average turning angle per individual cell (Fig- 
A

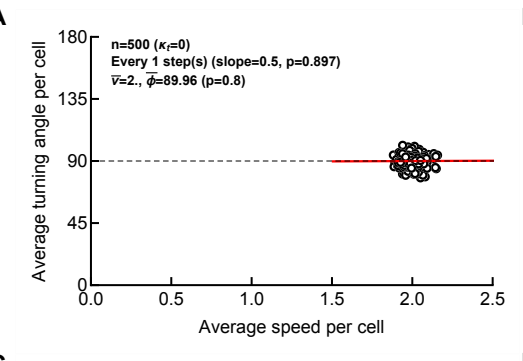

C

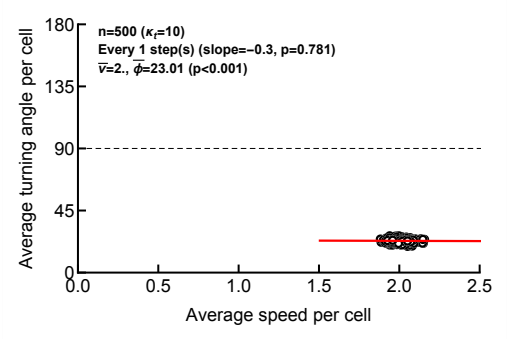

E

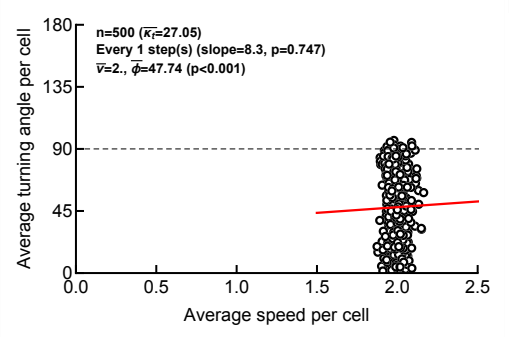

G

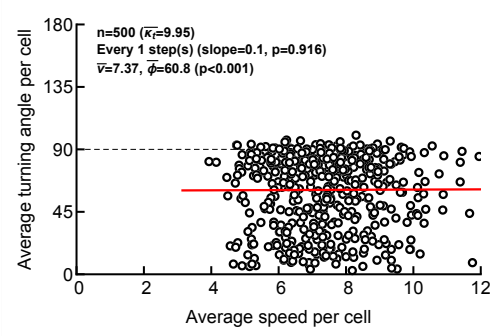

I

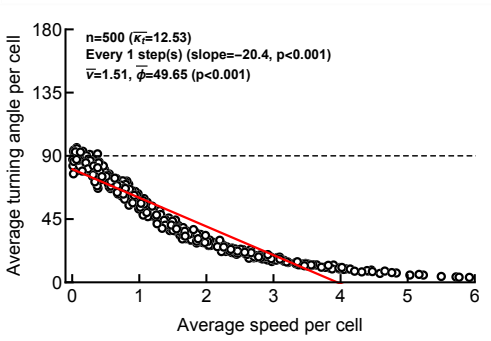

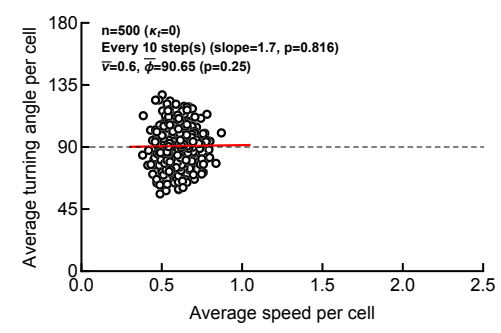
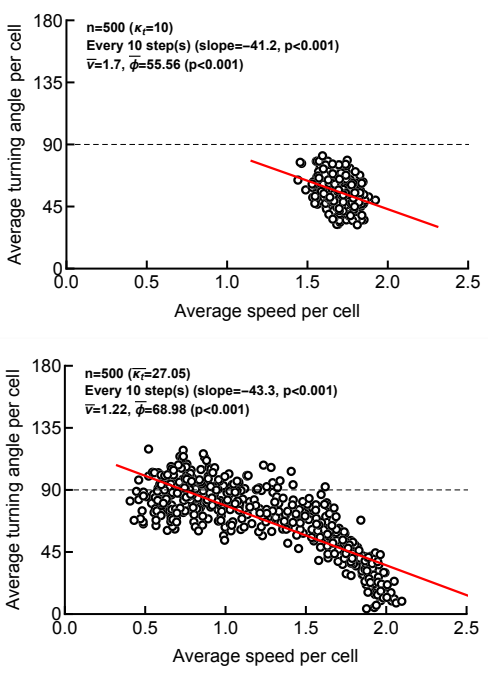

H

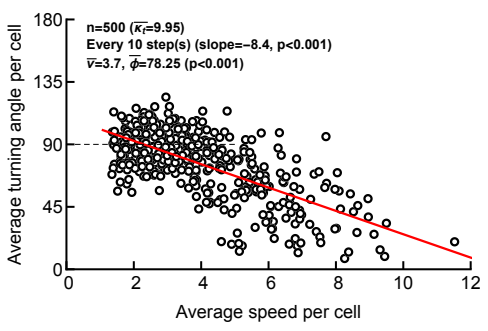

$\mathbf{J}$

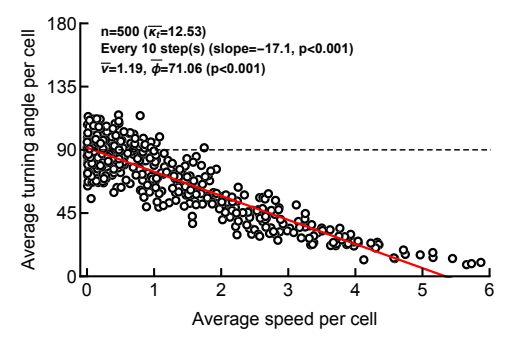

Figure 2: Correlation between average speed and average turning angle may arise in the absence of a cellintrinsic link between cells' speed and cells' turning angles due to sub-sampling. We simulated movement of 500 cells assuming i) Brownian walk $\left(\kappa_{t} \rightarrow 0, \mathrm{~A}-\mathrm{B}\right)$, ii) persistence for forward movement being identical for all cells $\left(\kappa_{t}=10, \mathrm{C}-\mathrm{D}\right)$, iii) heterogeneity in cells' persistence of movement ( $\kappa_{t}$ was sampled from a lognormal distribution, E-F), iv) independent heterogeneity in cells' persistence and speed movement $\left(\kappa_{t}\right.$ and $\bar{r}$ were sampled from a lognormal distribution); the resulting trajectories were sampled either every step (A,C,E,G) or every $k=10^{\text {th }}$ step (B,D,F,H). Other details of simulations are given in Materials and Methods. Each panel contains information on the average speed for all cells $(\bar{v})$, average turning angle for all cells $(\bar{\phi})$, and the result of linear regression of the average speed per cell and average speed per cell (denoted as "slope" and shown by red line) with $p$ value from the t-test. We also provide a test if the average turning angle of cells in the population is different from $90^{\circ}$ (Mann-Whitney test). We also simulated cell movements assuming a direct relationship between cells' persistence ability defined by $\kappa_{t}$ and cells' intrinsic movement speed $\left(\bar{r}=\ln \left(1+\kappa_{t}\right)\right.$, I-J). Note different scales in A-F and G-J due to higher speeds of cells in simulations in G-J. 
ure 2C-D). These simulations so far assumed that all cells in the population have the same intrinsic speed (determined by $\bar{r}$ ) and turning ability (determined by $\kappa_{t}$ ) which resulted in relatively small variability in estimated average speeds for individual cells due to random effects.

However, it is possible that cells differ in their ability to turn, e.g., either because of cell-intrinsic program or because of environmental constraints by physical barriers or chemical cues in specific locations of the tissue [11]. Therefore, in the third set of simulations we allowed every cell to have an intrinsic turning ability (defined by individual $\kappa_{t}$ ) drawn from a lognormal distribution (to allow for a broader range of $\kappa_{t}$ ). Importantly, while there was no correlation between speed and turning angle for frequently measured cell movements (Figure 2E), when sub-sampling we observed a strong negative correlation between speed and turning angle for a larger span of the speeds (Figure $2 \mathrm{~F}$ and see Supplemental figures S1-S3). Similar to "simpler" simulations, cells that had smaller a $\kappa_{t}$ had a higher propensity to turn, resulting in a smaller overall displacement, and thus, in smaller measured speeds. In contrast, cells that turn little (high $\kappa_{t}$ ) have estimated speeds close to the set intrinsic value $(\bar{r}=2)$. Interestingly, allowing for speeds to be a cell's property (i.e., when $\bar{r}$ for individual cells was sampled from a lognormal distribution) with random turning angles $\left(\kappa_{t} \approx 0\right)$ did not result in a negative correlation between speed and turning angle, suggesting that the ability to turn being cell-specific is the key to the observed correlation (Figure S4).

A

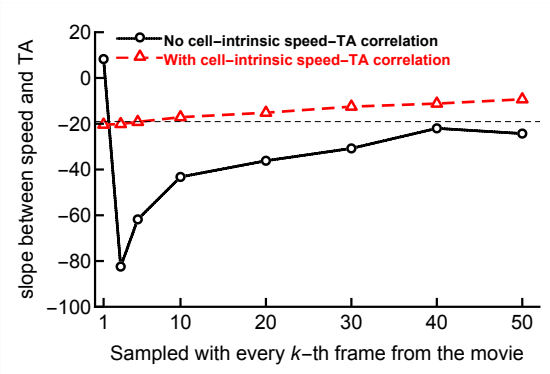

B

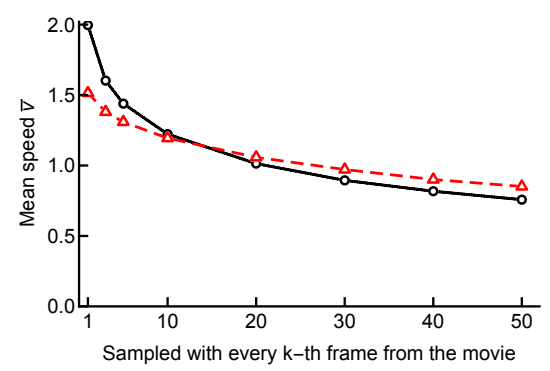

C

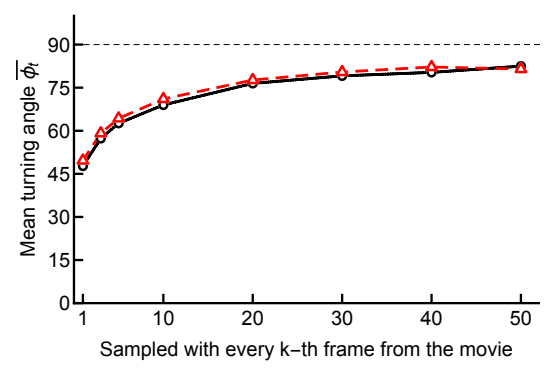

Figure 3: Frequency of imaging does not allow discrimination between hypotheses explaining negative correlation between measured average speed and average turning angle (TA) for a cell. We simulated movement of cells assuming that i) each cell in the population has a preference toward moving forward (defined by the concentration parameter $\kappa_{t}$ ) but all cells have the same intrinsic speed (Figure 2E-F) or ii) for each cell is characterized by a preference to move forward defined by $\kappa_{t}$ and intrinsic speed defined as $v^{(i)}=\ln \left(1+\kappa_{t}^{(i)}\right)$, where $(i)$ indicates an $i^{\text {th }}$ cell (Figure 2I-J). For different frequencies of recording cell movement, we calculated the slope between average speed and average turning angle (TA or $\bar{\phi}_{t}$ ) per cell (panel A), the average speed per cell $\left(\bar{v}\right.$, panel B), and the average turning angle per cell $\left(\bar{\phi}_{t}\right.$, panel $\left.\mathrm{C}\right)$ for the two hypotheses (without and with cell-intrinsic link between speed and turning angle, shown by different markers and lines). The thin dashed line in panel A denotes the expected slope for the model with a cell-intrinsic link between speed and turning at the lowest possible frequency of imaging $(-90 \arctan (\pi /(2 \bar{r})) /(\pi / 2) \approx-19$ for $\bar{r}=2)$. Values on the x-axes indicate which frames were included in the calculations. For example, $k=10$ indicates that data at $t=1,11,21,31 \ldots$ were used in calculations. In simulations, the concentration parameter $\kappa_{t}$ was lognormally distributed between cells with $\mu=1$ and $\sigma=2$ (see Figure 2 and Materials and Methods for more detail).

In the fourth set of simulations, we allowed both speed $(\bar{r})$ and persistence $\left(\kappa_{t}\right)$ to vary between individual cells independently. Using a lognormal distribution allowed for a range of speeds and average turning angles which were independent for frequently sampled data (Figure $2 \mathrm{G}$ ), but there was a strong negative correlation between average turning angle and average speed for coarsely sampled trajectories (Figure 2H and Figure S5) which resembled experimentally observed correlations (see below). 
Fifth and finally, we tested how the frequency of sampling of cell movements influences the observed correlation between cell speed and average turning angle when there is an intrinsic link between the instantaneous speed of the cell and its turning ability. We therefore simulated cell movement by sampling $\kappa_{t}$ from a lognormal distribution and then linked cells' intrinsic speed to cells' turning ability (by assuming $\bar{r}=\ln \left(1+\kappa_{t}\right)$ ). Interestingly, the frequency of sampling had a moderate effect on the negative correlation between average speed and average turning angle (Figure 2I-J and Figure S6). Taken together, these results strongly suggest that because the intrinsic cell speed, intrinsic turning ability, or frequency at which any cell makes decisions of turning are not known, a negative correlation between measured speed of cells and average turning angle need not arise from a cell-intrinsic program [18], but may be a simple consequence of coarse sampling.

Several measures such as average turning angle, speed, or MSD for cell cohorts do not allow to discriminate between alternative hypotheses. In our simulations it was clear that the frequency of sampling had a major impact on the regression slope between average cell speed and average turning angle (e.g., Supplemental figures S1-S3). We therefore tested if a change in the frequency of sampling of cell movement can be used to discriminate between cell-intrinsic vs. randomly arising negative correlation between speed and turning angle. We calculated how the slope between speed and turning angle, average speed, and average turning angle change when the frequency of imaging changes (Figure 3). Unfortunately, the two models were similar in how these parameters changed with imaging frequency, except for the narrow range when imaging frequency would coincide with the frequency at which cells make turn decisions (Figure 3A at $k=1$ ). However, very frequent imaging contains many artifacts due to changes in cell shape or fluorescence signal from the cell [26], suggesting that changes in the frequency of imaging may not be the method to discriminate between these alternatives.

The mean square displacement (MSD) plots have been also used to establish the linear relationship between persistence and speed for cohorts of cells that have similar speeds [18]. Specifically, cell cohorts with higher speeds had a faster increase in MSD with time (higher $\gamma$, see eqn. (5) and other details in Materials and methods) that was interpreted as evidence of the direct relationship between cell speed and persistence [18]. We therefore performed additional analyses to determine if we can reject the null model in which turning ability and speeds are cell-dependent but are uncorrelated using this methodology. For this we simulated movements of 500 cells assuming correlated random walks using the von Mises-Fisher distribution. In one set of simulations, we considered the persistence $\kappa_{t}$ and the speed characterizing parameter $\bar{r}$, drawn from two independent lognormal distributions (Figure 4A-B and Figure S7). As expected, the frequency of sampling impacted dramatically how different cell cohorts displaced over time (Figure S7), and while for frequent (every $k=1$ frame) sampling different cell cohorts were similarly super-diffusive with $\gamma>1$ (Figure 4A and Figure S7A), coarse sampling (every $k=10$ frames) resulted in different cohorts displacing differently, with slowest cohorts having low $(\gamma=1.2$, Figure $4 \mathrm{~B})$ or close to Brownian diffusion $(\gamma \approx 1$, Figure S7D).

In another set of simulations, we let the persistence $\kappa_{t}$ be drawn from a lognormal distribution with $\bar{r}$ being directly determined by $\kappa_{t}$ through the relation $\bar{r}=\ln \left(1+\kappa_{t}\right)$. As expected, frequent sampling (every movement, $k=1$ ) resulted in cell cohorts with different speeds displaying different rates of displacement, with slower cells displacing nearly as Brownian $(\gamma \approx 1$, Figure $4 \mathrm{C})$. Importantly, for coarsely sampled data $(k=10)$, displacement of cell cohorts with different speeds did not dramatically change (Figure 4D), and was similar to the simulations in which speed and persistence were not intrinsically correlated (compare Figure 4B \& 4D). This further suggests that MSD plots for cell cohorts with different speeds cannot be used to reject the null model and strengthens our argument 
A

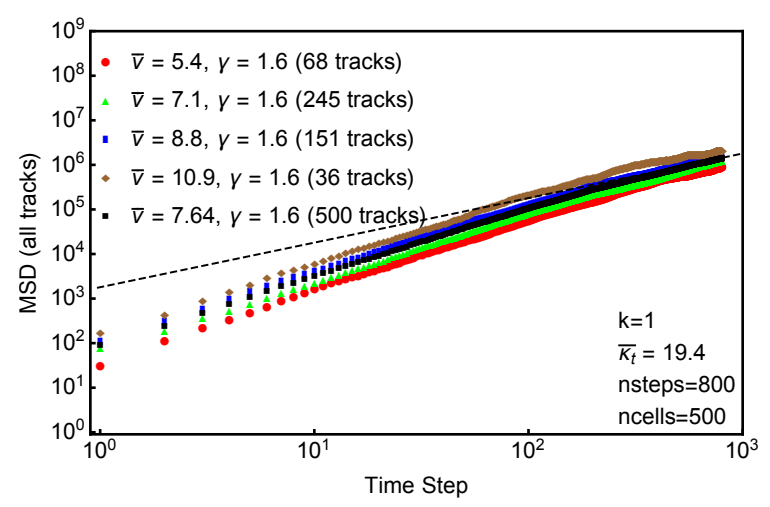

C

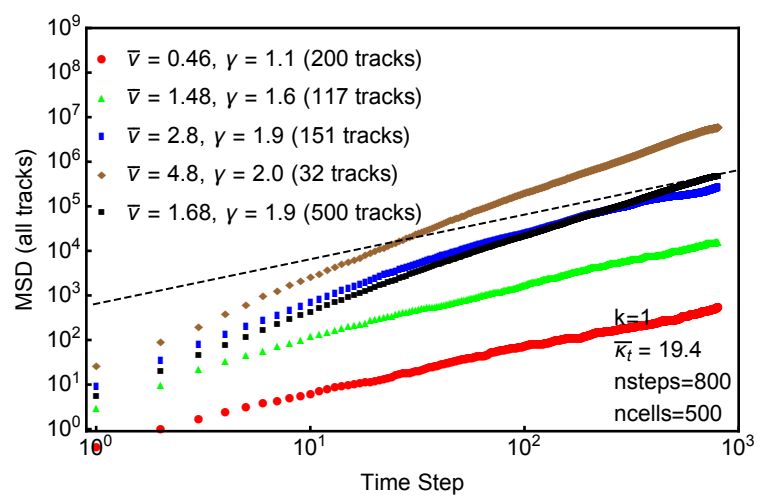

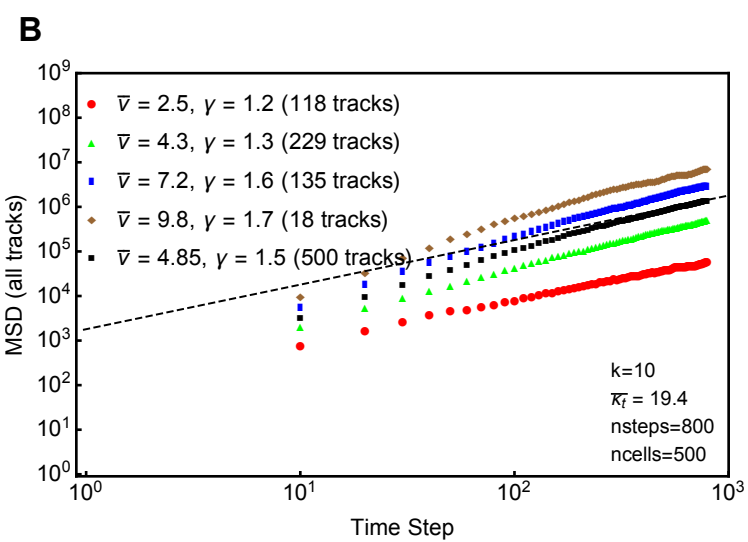

D

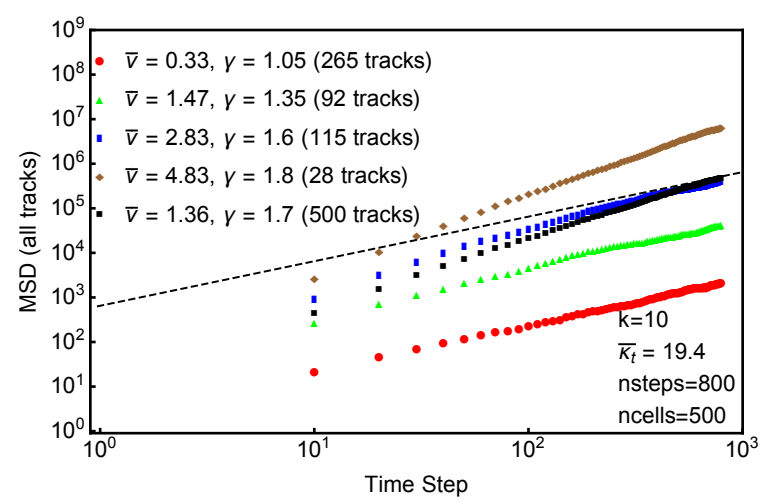

Figure 4: Change in mean square displacement (MSD) with time for different cell cohorts is qualitatively equivalent whether there is or there is not an intrinsic link between the average speed and average turning angle per cell under coarse sampling of the cell trajectories. We simulated movement of cells assuming a correlated random walk characterized by the concentration parameter $\kappa_{t}$ of the VMF distribution (see eqn. (1)) and an independent distribution of cell speeds (characterized by the mean $\bar{r}$ of the Pareto distribution, see eqn. (3); panels $\mathrm{A} \& \mathrm{~B}$ ) or when there is a direct correlation between $\kappa_{t}$ and cell speed (panels C\&D). We sampled the movement data either every step $(k=1, \mathrm{~A} \& \mathrm{C})$ or even $10^{\text {th }}$ step $(k=10, \mathrm{~B} \& \mathrm{D})$. In all simulations, the parameter $\kappa_{t}$, which dictates the average turning angle of a cell (i.e., persistence), is randomly drawn from a log-normal distribution with mean $\mu=1$ and standard deviation $\sigma=2$ (eqn. (4)). The timestep indicates the regular intervals at which we simulated the cell positions, and MSD is dimensionless. In one set of simulations (A-B), we randomly draw $\bar{r}$ from an independent log-normal distribution with mean $\mu=2$ and standard deviation $\sigma=0.2$. In the Pareto distribution we set $\alpha=3$ for every cell we calculated $r_{\min }=\bar{r}(\alpha-1) / \alpha$. In another set of simulations, we let $\bar{r}=\ln \left(1+\kappa_{t}\right)$ for every cell (C\&D). Simulations were done with $n=500$ cells for 800 timesteps. Four speed bins are considered from the distribution of average speed per cell and for each bin MSD is computed. We also include the MSD for all tracks together. To characterize the MSD we used the relation MSD $=c t^{\gamma}$ where $\gamma=1$ suggests Brownian diffusion (denoted by a thin black dashed line) and $\gamma>1$ suggests superdiffusion. The parameter $\gamma$ was estimated for each MSD curve by linear regression of the $\log -\log$ transformed MSD data. 
that the observed correlation between average cell speed and average turning angle, or in general any observed correlation between cell parameters that describes the motility need not necessarily arise from a cell-intrinsic behavior hardwired within the cells.

So far our simulations did not allow to find a significant difference in several major characteristics of cell movement between model with an intrinsic link between cell persistence and speed and a model without such link. We therefore wondered if comparing simulations to actual data may allow to see the inadequacy of the "sub-sampling" model. Comparing the model simulations with the data was not trivial, however, because our simulations were done using dimensionless units (time step, movement length) and the data have dimensions, and which specific details of the data need to be compared with simulations may be debated. We opted for a simple approach whereby we attempt to reproduce the experimentally observed correlation between average turning angle per cell and average speed with that found in simulations. For a fair comparison we needed the data to be sampled at regular time intervals but we found that in about $35 \%$ of tracks in control experiments of Jerison \& Quake [18] (out of 712) there were missing time step values. We therefore cleaned the data splitting the tracks with missing values and assigning new track ID to each of the new trajectories [11]. This resulted in 1337 trajectories with now identically spaced measurements (every 45sec) with 68 movements per track on average. For every trajectory we calculated the average speed and average turning angle and found a strong negative correlation between the two parameters (Figure 5 and Figure S8).

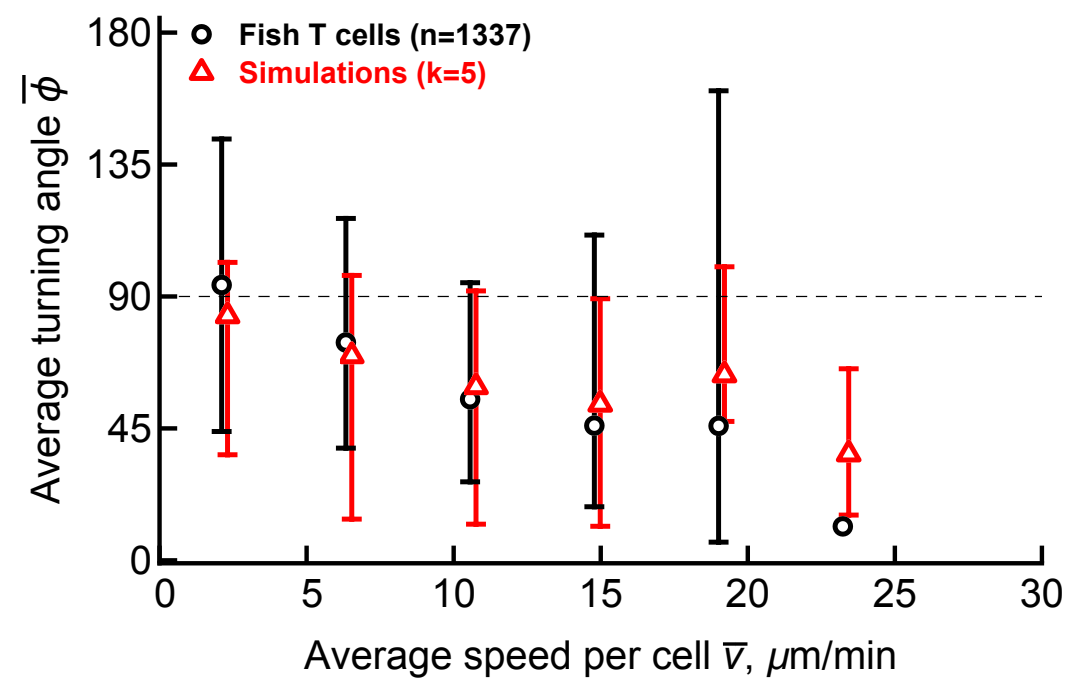

Figure 5: Matching simulations of cell movement in which cell's speed and turning ability are uncorrelated with experimental data. For cleaned data of Jerison \& Quake [18] we calculated the average turning angle and speed for every trajectory and plotted binned data. Note that the bin with largest speed had only one trajectory. We also performed simulations in which every cell has a defined persistence ability and speed, sub-sampled the resulting simulation data (every $k=5$ th step was used, see Figure S8 for more detail). To simplify calculation we assumed that this sampling frequency is $1 \mathrm{~min}$, calculated the average turning angle and average speed for every trajectory, and then binned these simulation data in the identical way to that of actual experimental data. Confidence intervals denote 2.5 and 97.5 percentiles of the data. Also note that while experimental data were collected in 2D by ignoring z-coordinate of the moving cells [18], our simulations were done in 3D.

We then performed a series of simulations with 1000 cells each undergoing 100 movements by 
varying distribution of persistence ability (determined by concentration $\kappa_{t}$ ) and speed (determined by the average movement length $\bar{r})$ and sub-sampling frequency $(k>1)$. Then for every track in the simulated data we calculated the average speed and average turning angle and binned those data into cell cohorts with similar average speeds as we did for experimental data (Figure S8). We found that many different parameter combinations provide a reasonable match of the experimental data (e.g., Figure 5) further suggesting that a model in which turning ability of $\mathrm{T}$ cells and their intrinsic speed are uncorrelated is consistent with experimental data.

Experimentally detected correlation between speed and turning depends on the imaging frequency. Given that the correlation between average turning angle and average speed of a cell in the population may arise due to sampling, we finally investigated how sampling of cell trajectories in actual cell movement data may impact this correlation. Using previously published data of movement of T cells in zebrafish from Jerison \& Quake [18], we calculated the average turning angle and average speed of every $\mathrm{T}$ cell in the control dataset containing $n=712$ cells and found a strong negative correlation between these parameters (Figure 6A). Interestingly, reducing the sampling rate to every 10 frames (or from every $45 \mathrm{sec}$ to every $450 \mathrm{sec}=7.5 \mathrm{~min}$ ) made the correlation non-significant (Figure 6C), and even resulted in a weak but statistically significant positive correlation at coarser sampling (Figure 6D). While this result may be expected given that $\mathrm{T}$ cells undergoing a correlated random walk should be eventually turning their trajectories, the loss of correlation between persistence and speed at sub-sampling suggests that the strength of this correlation is weak.

Jerison \& Quake [18] also performed experiments in which T cells were imaged at a higher frequency, every $12 \mathrm{sec}$. We found that in these data, sub-sampling at every 20 frames (i.e., every $240 \mathrm{sec}=4 \mathrm{~min}$ ) also resulted in the loss of statistically significant negative correlation between average turning angle and average speed (results not shown). Interestingly, higher frequency imaging resulted in higher average speeds of cells, which was associated with lower turning angles (Figure S9A-D); both of these observations are fully consistent with the model in which there is uncorrelated heterogeneity in cell speed and turning ability (e.g., Figure S5). Treating zebrafish with the drug that impedes movement of $\mathrm{T}$ cells resulted in slower $\mathrm{T}$ cell speeds and larger turning angles (Figure S9E-F), which again is consistent with the "null" model. Taken together, our results suggest that current experimental observations are fully consistent with the hypothesis that the observed correlation between cell persistence and speed arises due to sub-sampling, and thus challenges the novel paradigm that there are fundamental biological mechanisms that simultaneously regulate intrinsic speeds and persistence of cells.

\section{Discussion}

Cell migration is a complicated process. In general cells move randomly, often by correlated random walks as determined by the turning angle and movement length distributions [11]. Whether there are basic fundamental principles that determine movement strategies of cells remains debated. Recent studies with various cell types, conditions, and constraints (e.g., genetic mutations) have shown that faster cells tend to move straighter (i.e., more persistently) and slower cells tend to change direction more often, resulting in a positive correlation between persistence time and cell speed, or equivalently, in a negative correlation between average turning angle and average speed per cell [18, 23, 24, 26]. The generality of this correlation across multiple cell types and conditions is a strong argument that the correlation arises due to a fundamental, cell-intrinsic movement program that is conserved across 
A

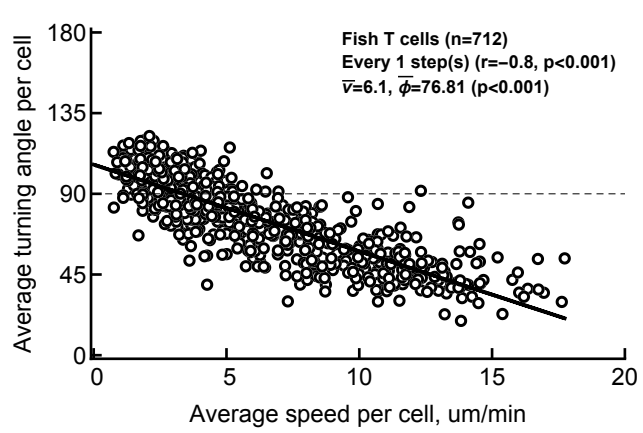

C

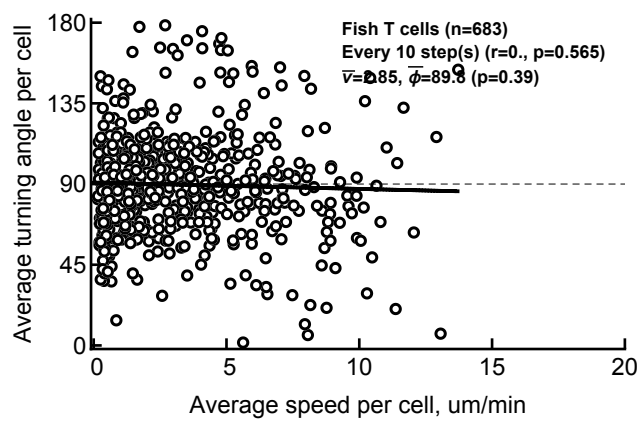

B

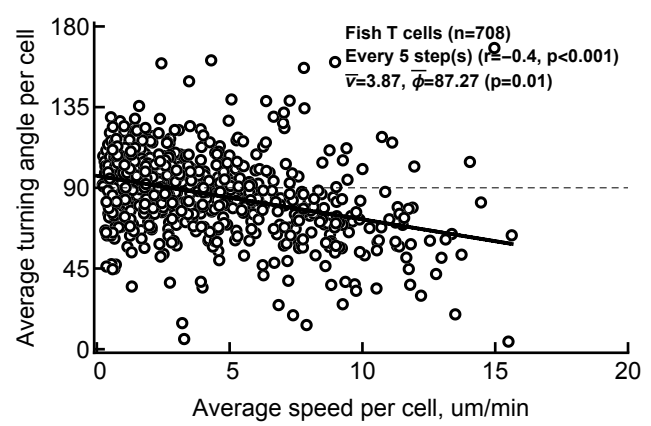

D

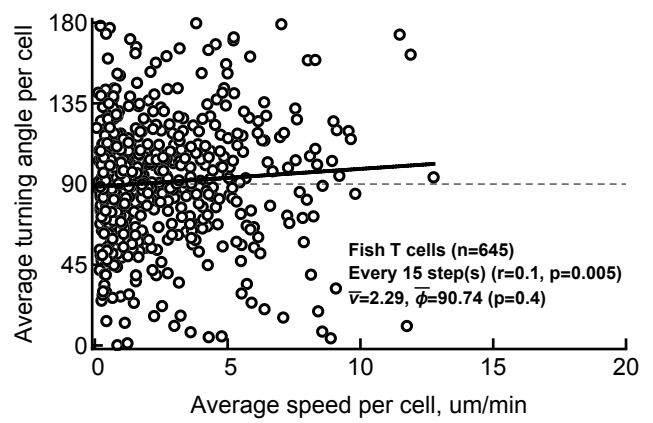

Figure 6: Negative correlation between average speed and average turning angle for individual cells in experimental data disappears when the data are coarsely sampled. For the previously published experimental data from Jerison \& Quake [18] (selected as "control" experiments with "fish T cells", $n=712$ tracks in total) we calculated the average speed and average turning angle for every cell/track assuming that sampling occurs every $k$ steps with $k=1$ (panel A), $k=5$ (panel B), $k=10$ (panel C), and $k=15$ (panel D). In particular, when $k=10$, only cell positions at times $t=1,11,21, \ldots$ are included in calculating average speed and average turning angle. Note that for coarse sampling, some tracks are not included because they have fewer than $3 k$ measurements precluding calculating a single turning angle. However, results were similar if we only track $n=645$ cells with long enough tracks for sampling every $k=15^{\text {th }}$ cell position (results not shown).

different systems [18, 23]. Here we argue that because both turning angles and speeds are measured parameters, they may be sensitive to the frequency at which movement of cells is recorded. Assuming that cells in a given population have a broad and uncorrelated distribution of intrinsic movement speeds and turning abilities naturally results in a negative correlation between average turning angle and average speed when sampling of cell trajectories is done less frequently than the cell's "decision" for turning. This simple (null) hypothesis also explains the universality of the negative correlation between turning angle and speed for different cell types and various conditions: because all such data are generated using similar imaging techniques that are prone to sub-sampling. Our model also makes a testable prediction that negative correlation between speed and turning may be observed in other systems, e.g., in animal movement.

While re-analyzing the $2 \mathrm{D}$ cell track data for $712 \mathrm{~T}$ cells in zebrafish in the "control" category [18], we found that a strong negative correlation between average turning angle and speed disappears if the data are subsampled (Figure 6). This suggests that a cell-intrinsic link between cell speed and persistence, if it exists, is not extremely strong. In our simulations we found that in the presence of cell-intrinsic correlation between cell speed and persistence (defined by $\kappa_{t}$ ), sub-sampling does not remove the correlation between average turning angle and average speed; however, this result may depend on assumed model parameters (results not shown). The frequency of imaging impacts the measured speeds and distribution of turning angles for T cells in zebrafish (Figure S9A-D) highlighting 
the difficulty in comparing results from different studies that used different imaging settings.

We found that simulating cell movement assuming that there is or not a link between persistence and speed generated very similar movement characteristics, for example, change in MSD vs. time (Figure 4). Furthermore, average turning angle or average speed were similar in two models when sampling rate was changed (Figure 3). Interestingly, we could relatively well match experimentally observed correlation between average turning angle and average speed per cell using simulations (Figure 5). However, there is one important consequence of this result. To do such a match we had to assume the actual dimension value for the frequency of sub-sampling. Specifically, to produce the results in Figure 5 we assumed that sampling occurs at every minute (so that movement lengths calculated in simulations are then the cell speed in $\mu \mathrm{m} / \mathrm{min})$. This implies that actual cell movements occur at every $12 \mathrm{sec}(k=5)$, and for such movements, the average movement length is $\bar{r}=8.3 \mu \mathrm{m}$. Whether cells are able to make average movements at speeds of $8.3 / 12=41.5 \mu \mathrm{m} / \mathrm{min}$ and what is the maximal speed that $\mathrm{T}$ cells (or other cells) can exhibit has not been rigorously investigated.

In our analysis of the data on movement of $\mathrm{T}$ cells in zebrafish we noticed an interesting peculiarity: the unusual distribution of turning angles for the population of cells with most cells (37\%) have extremely low turning angles $\left(<45^{\circ}\right)$, and many other cells $(26 \%)$ having sharp, turning-back angles $\left(>135^{\circ}\right.$, Figure S9A). A similar distribution of turning angles was observed for $\mathrm{T}$ cells moving in collagen-fibrin gel that was recorded in $2 \mathrm{D}$ settings [16]. In contrast, for $\mathrm{T}$ cells moving in lymph nodes or the liver the distribution of turning angles is lognormal-like, with the dominant peak at acute, but moderate turning angles (see Figures S2A and S7E in [11]). We speculate that this is because in studies by Rajakaruna et al. [11], movement of $\mathrm{T}$ cells was recorded with two photon microscope providing 3D coordinates of cells while these previous studies did not take 3D coordinates into account. In support of this hypothesis we did find that ignoring the $z$ coordinate in our data for $\mathrm{T}$ cells walks in the liver or LNs resulted in more cells with smaller turning angles, but we did not see a large increase in cells turning back (results not shown). Therefore, it is possible that movement of $\mathrm{T}$ cells in zebrafish is different than that in mice.

Our work has several limitations. The major limitation we believe is that we could not come up with an experimental or computational way to reject the null model in which the negative correlation between average turning angle and average speed arises due to sub-sampling of trajectories of heterogeneous cells. This remains a challenge for future studies. Moreover, how sampling (suband over-sampling) contributes to the observed patterns of cell movement remains to be investigate more rigorously, and studies that can estimate the "speed-of-light" of cells (i.e., maximal speed that cells can actually have) would be important to constrain impact of sub-sampling on inferred speeds of cells. Studies measuring kinetic details of cell migration suggested that actin, the major protein involved in cell movement, can polymerize at a rate of $5 \mu \mathrm{m} / \mathrm{s}=300 \mu \mathrm{m} / \mathrm{min}$ [27]. Therefore, the potential upper limit of cell's instantaneous speed is unlikely to exceed this limit.

Another limitation is the relatively arbitrary choice of model parameters, such as distributions of turning angles (characterized by the concentration parameter of the von Mises-Fisher distribution) and the distribution of speeds (characterized by the Pareto distribution) and their relationship to the frequency at which movements of simulated cells are sampled. Our results suggest that the negative correlation between turning angle and speed should be observed for many parameter combinations and we could find some sets of parameters with which we could match experimentally observed correlation. However, whether our assumed distributions are realistic remains unknown and it is likely that our assumption and every cell in the population has a fixed "persistence" ability is an 
oversimplilfication. Rather, ability of cells to persist (or turn) can be determined by environmental constrains and/or cues [11], and thus may change over time. Indeed, in contrast with experimental data which sub-sampling results in loss of correlation between average turning angle and speed (Figure 6 ), in simulations sub-sampling does not suggesting that movement persistence is not constant over time for individual cells.

Finally, we have used our novel approach, based on von Mises-Fisher distribution, to simulate correlated random walks [25]. While theoretically straightforward, properties of cells moving in accord to VMF distribution may not fully correspond to the more commonly used approach, the Orenstein-Uhlenbeck (O-U) model, which has a well-defined infinitesimal limit [18, 28]. Determining similarities and differences in cell trajectories generated using the VMF distribution or the O-U model is the focus of our current research.

Given that current studies are apparently insufficient to reject the null model, an important philosophical question is whether it is required to design additional experiments (which will cost time and money) to reject the null model in favor of the model assuming the existence of a cellintrinsic link between persistence of a cell and its speed. Future studies need to investigate if the presence of the cell-intrinsic link between persistence and speed impacts predictions of mathematical models on, for example, efficiency of T cell search for infection [25].

Movement persistence, the propensity of moving cells to keep forward movement when environment and conditions are constant, can be postulated as a type of "biological inertia" (or "biological conservation of momentum") specific to the cells [11]. Per such a postulate, changes in the environment (e.g., shape of the surface on which cells move, cues, or change in nutrients) are responsible for cells changing movement, e.g., to stop and/or turn. It should be noted, however, that such "biological conservation of momentum" or "inertia" should not be confused with physical inertia, because viscous forces are much stronger than the inertial forces for biological (small) cells (the Reynolds number is of the order of $10^{-4}$, which classifies the cell movements under physical processes of low Reynolds numbers [29]). A more integrated experimental approach is needed to be capable of examining continuous cell movements as we have observed in some experiments [15]. One of the major shortcomings of our study is that we could not come up with a proposed experimental design that might rule out one of the alternative hypothesis that we discussed. Future studies should nevertheless attempt to define ways to falsify the simpler explanation of observed negative correlation between cell speed and turning angle as the consequence of sub-sampling.

\section{Materials and Methods}

Choosing turning angles using von Mises-Fisher distribution. To simulate cell movement in 3D, we assumed that cells undergo a correlated (persistent) random walk with the degree of persistence determined by the concentration parameter $\kappa_{t}$ in the von Mises-Fisher (VMF) distribution $[25,30]$, which is a probability distribution on an $n$-dimensional sphere (in our case, $n=3$ ) that chooses a direction with measurable bias toward a given direction. The distribution is

$$
P\left(\chi \mid \mu, \kappa_{t}\right)=\frac{\kappa_{t} \mathrm{e}^{\kappa_{t} \mu^{T} \chi}}{2 \pi\left(\mathrm{e}^{\kappa_{t}}-\mathrm{e}^{-\kappa_{t}}\right)},
$$


where $\mu$ is the direction vector toward which there is bias (e.g., the previous movement vector), $\chi$ is the newly chosen direction vector, $\kappa_{t}$ is the concentration (with 0 meaning no bias, positive meaning persistence, and negative meaning aversion), and $|\mu|=|\chi|=1$. Random (biased) vectors given direction $\mu$ and concentration parameter $\kappa_{t}$ were generated in Mathematica (v. 12.1) by choosing a vector with bias toward direction $\{0,0,1\}$, which simplifies the process to choosing 1) $x$ and $y$ randomly from a normal distribution $N(0,1)$ (using command RandomVariate), and 2) $z$ based on the von Mises-Fisher distribution, chosen by

$$
z=1+\left(\ln (r)+\ln \left(1+(1-r) \frac{e^{-2 \kappa t}}{r}\right)\right) / \kappa_{t}
$$

where $r$ is chosen uniformly between 0 and 1 [31,32]. Then $x$ and $y$ are weighted to place the chosen vector on the unit sphere, and then we use a rotation transform (command RotationTransform) to adjust the generated vector with respect to the desired bias direction. The native Mathematica code to generate a random vector using the VMF distribution is

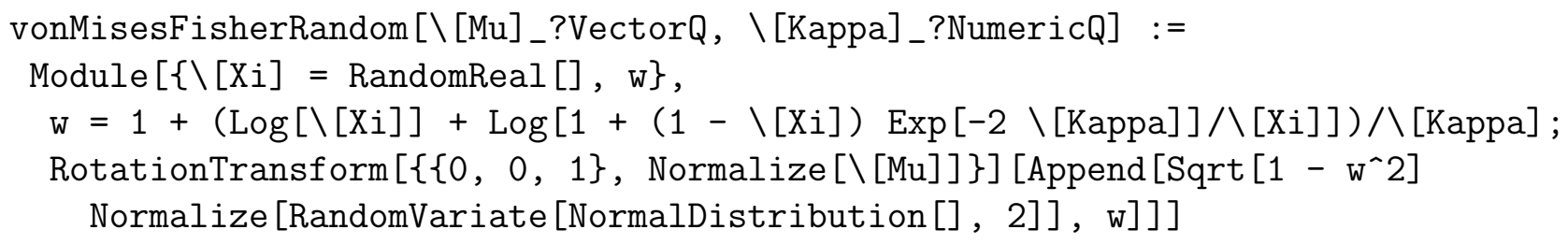

Choosing movement length distribution. The length of the movement was drawn randomly from the Pareto (powerlaw) distribution

$$
f\left(r \mid r_{\min }, \alpha\right)=\frac{\alpha r_{\min }^{\alpha}}{r^{\alpha+1}}
$$

where $r_{\min }$ and $\alpha$ are the scale and shape parameter, respectively, and $\bar{r}=\alpha r_{\min } /(\alpha-1)$. In the Pareto distribution, $r \geq r_{\min }$. In simulations, we assumed $\alpha=5.5$, corresponding to a Brownian-like distribution of movement lengths [11], $\bar{r}=2$, and $r_{\min }=\bar{r}(\alpha-1) / \alpha$. Thus $\kappa_{t}$ determines the degree of walk persistence (i.e., the average turning angle) and $r_{\min }$ and $\alpha$ determine the speed of the cell movement. As these quantities are independent, in most of our simulations speed and turning angles truly have no correlation.

Simulation details. In simulations, each cell moves in a random direction (determined by $\kappa_{t}$ in eqn. (1) and by the previous movement vector) and by a random distance (determined by $r_{\min }$ and $\alpha$ in eqn. (3)). However, if cell movements are measured at a lower frequency than the cell is moving, then the measured cell movement speed and average turning angles are calculated from the "assumed" trajectory that takes into account only some of the cell's positions. For example, in simulating cell movement for 100 steps where we only count every $k=10^{\text {th }}$ step as a movement, we calculated instantaneous speeds and turning angles by taking positions $1,11, \ldots 91$ and then calculating the average speed $\bar{v}$ and average turning angle $\bar{\phi}_{t}$ per cell using these positions by dividing the distance travelled by the cell at these time points by $k$. This approach allows to properly compare mean speeds for different sub-sampling rates $k$. 
In some simulations, we assumed that every cell has an inherent concentration parameter $\kappa_{t}$ which determines cells' persistence ability. We sampled values of $\kappa_{t}$ from a lognormal distribution defined as

$$
p\left(\kappa_{t} \mid \mu, \sigma\right)=\frac{1}{\sqrt{2 \pi} \kappa_{t} \sigma} \mathrm{e}^{-\frac{\left(\ln \left(\kappa_{t}\right)-\mu\right)^{2}}{2 \sigma^{2}}},
$$

with typically chosen $\mu=1$ and $\sigma=2$ (but see Main text for other examples).

We also simulated cell movement with the intrinsic cell movement speed (determined by $\bar{r}$ ) and persistence in the walk (determined by $\kappa_{t}$ ) being correlated. We sampled $\kappa_{t}$ for each cell from a lognormal distribution (eqn. (4)) with parameters $\mu=1$ and $\sigma=2$ and let the average movement length for each cell be $\bar{r}=\ln \left(1+\kappa_{t}\right)$. Then, setting $\alpha=5.5$, we let for every cell $r_{\min }=\bar{r}(\alpha-1) / \alpha$ in eqn. (3). Movement length distribution was then sampled randomly from Pareto distribution with $r_{\min }$ and $\alpha$ using RandomVariate function in Mathematica.

When simulating a distribution of cell-intrinsic speeds we assumed that for each cell, $\bar{r}$ in the Pareto distribution follows a lognormal distribution as for $\kappa_{t}$ (eqn. (4)) with $\mu=1$ and $\sigma=2$ (and $\alpha=5.5)$.

Mean squared displacement. For the computation of the mean square displacement (MSD), we used the relation

$$
\operatorname{MSD}(t)=\frac{1}{N} \sum_{i=1}^{N}\left|\overrightarrow{r_{i}}(t)-\overrightarrow{r_{i}}(0)\right|^{2}
$$

where $\overrightarrow{r_{i}}(t)$ is the position vector of the cell $i$ at time $t$ and $\overrightarrow{r_{i}}(0)$ is the initial position and $N$ represents the total number of cell tracks considered (typically in our simulations $N=500$ ). For different cell cohorts, we divided the total cell tracks into speed groups based on the average speed distribution of the individual cell tracks. To characterize the MSD plots we fitted a relation $\operatorname{MSD}(t) \propto t^{\gamma}$ to estimate $\gamma$ for all cells or cohorts of cells with similar movement speeds. Specifically, we log-transformed MSD and time, fitted a line to the transformed data, and estimated the slope $\gamma$. Note that $\gamma=1$ represents normal (Brownian) diffusion and $\gamma>1$ represents super-diffusion.

Parameter dimensions and relating simulations to data. It should be noted that in our simulations we did not choose dimensions of the parameters, so the time units are in steps $(1,2,3$, etc) and movement length units are also dimensionless. This was done to prevent a bias towards particular numerical values which may differ between different biological systems. Therefore, estimated quantities such as average speed per cell has a dimension of displacement unit per time unit. To link simulation results to experimental data we assumed that when we sub-sample the data at rate $k$ (i.e., when $k=10$ every 10 th movement is only used), the calculated average speed per cell is then given in $\mu \mathrm{m} / \mathrm{min}$ units which effectively assumes that we sample movements every $d t=1 \mathrm{~min}$, so the actual movements by cells occur at a rate $d t / k$. 


\section{Data sources}

Data presented in Figure 6 and Figure S9 were provided by Jerison \& Quake [18] and are available via a link to github in the original publication.

\section{Code sources}

All analyses have been primarily performed in Mathematica (ver 12) and codes used to generate most of figures in the paper are provided on GitHub:

https://github.com/vganusov/correlated_random_walk_simulations [33].

\section{Ethics statement}

No animal or human experiments performed.

\section{Author contributions}

The question for the study arose during discussions of the cell movement data between all authors. VVG ran the simulations using methods by VSZ and discussed results with other authors. BM wrote the first draft of the paper. BM, VVG, and VSZ contributed to the final draft of the paper.

\section{Acknowledgments}

We would like to thank E. Jerison and S. Quake for the valuable discussions of our results and comments on the previous versions of the paper. This work was supported by the NIH grant (R01 GM118553) to VVG.

\section{References}

1. Lauffenburger, D. A., Rivero, M., Kelly, F., Ford, R. \& DiRienzo, J. 1987 Bacterial chemotaxis. Cell flux model, parameter measurement, population dynamics, and genetic manipulation. Annals of the New York Academy of Sciences, 506, 281-295. doi:10.1111/j.1749-6632.1987.tb23827.x.

2. King, J. S. \& Insall, R. H. 2009 Chemotaxis: finding the way forward with Dictyostelium. Trends in cell biology, 19, 523-530. doi:10.1016/j.tcb.2009.07.004. 
3. Cheng, Y., Felix, B. \& Othmer, H. G. 2020 The roles of signaling in cytoskeletal changes, random movement, direction-sensing and polarization of eukaryotic cells. Cells, 9. doi: 10.3390/cells9061437.

4. De la Fuente, I. M. \& Lopez, J. I. 2020 Cell motility and cancer. Cancers, 12 doi: $10.3390 /$ cancers12082177.

5. Gaylo, A., Schrock, D. C., Fernandes, N. R. J. \& Fowell, D. J. 2016 T cell interstitial migration: Motility cues from the inflamed tissue for micro- and macro-positioning. Frontiers in immunology, 7, 428. doi:10.3389/fimmu.2016.00428.

6. Krummel, M. F., Bartumeus, F. \& Gerard, A. 2016 T cell migration, search strategies and mechanisms. Nature reviews. Immunology, 16, 193-201.

7. Miller, M. J., Wei, S. H., Cahalan, M. D. \& Parker, I. 2003 Autonomous t cell trafficking examined in vivo with intravital two-photon microscopy. Proceedings of the National Academy of Sciences, 100(5), 2604-2609.

8. Textor, J., Peixoto, A., Henrickson, S. E., Sinn, M., von Andrian, U. H. \& Westermann, J. 2011 Defining the quantitative limits of intravital two-photon lymphocyte tracking. Proceedings of the National Academy of Sciences, 108(30), 12 401-12406.

9. Germain, R. N., Robey, E. A. \& Cahalan, M. D. 2012 A decade of imaging cellular motility and interaction dynamics in the immune system. Science, 336(6089), 1676-1681.

10. Ariotti, S., Beltman, J. B., Chodaczek, G., Hoekstra, M. E., Van Beek, A. E., Gomez-Eerland, R., Ritsma, L., Van Rheenen, J., Marée, A. F. et al. 2012 Tissue-resident memory cd8+ t cells continuously patrol skin epithelia to quickly recognize local antigen. Proceedings of the National Academy of Sciences, 109(48), 19 739-19744.

11. Rajakaruna, H., O’Connor, J., Cockburn, I. A. \& Ganusov, V. V. 2020 Environment-imposed constraints can make brownian walkers efficient searchers. bioRxiv. doi:10.1101/2020.11.06.371690.

12. Mrass, P., Oruganti, S. R., Fricke, G. M., Tafoya, J., Byrum, J. R., Yang, L., Hamilton, S. L., Miller, M. J., Moses, M. E. et al. 2017 ROCK regulates the intermittent mode of interstitial T cell migration in inflamed lungs. Nature communications, 8(1), 1-14.

13. Harris, T. H., Banigan, E. J., Christian, D. A., Konradt, C., Wojno, E. D. T., Norose, K., Wilson, E. H., John, B., Weninger, W. et al. 2012 Generalized Lévy walks and the role of chemokines in migration of effector CD8+ T cells. Nature, 486(7404), 545-548.

14. Novkovic, M., Onder, L., Cupovic, J., Abe, J., Bomze, D., Cremasco, V., Scandella, E., Stein, J. V., Bocharov, G. et al. 2016 Topological small-world organization of the fibroblastic reticular cell network determines lymph node functionality. PLoS biology, 14, e1002515. doi: 10.1371/journal.pbio.1002515.

15. McNamara, H. A., Cai, Y., Wagle, M. V., Sontani, Y., Roots, C. M., Miosge, L. A., O'Connor, J. H., Sutton, H. J., Ganusov, V. V. et al. 2017 Up-regulation of LFA-1 allows liver-resident memory $\mathrm{T}$ cells to patrol and remain in the hepatic sinusoids. Science Immunology, pp. 1-10.

16. Wu, P.-H., Giri, A., Sun, S. X. \& Wirtz, D. 2014 Three-dimensional cell migration does not follow a random walk. Proceedings of the National Academy of Sciences, 111(11), 3949-3954. doi:10.1073/pnas.1318967111. 
17. Wu, P.-H., Giri, A. \& Wirtz, D. 2015 Statistical analysis of cell migration in 3D using the anisotropic persistent random walk model. Nature protocols, 10, 517-527. doi: 10.1038/nprot.2015.030.

18. Jerison, E. R. \& Quake, S. R. 2020 Heterogeneous T cell motility behaviors emerge from a coupling between speed and turning in vivo. eLife, 9. doi:10.7554/eLife.53933.

19. Beltman, J. B., Marée, A. F. M. \& de Boer, R. J. 2009 Analysing immune cell migration. Nat Rev Immunol, 9(11), 789-798.

20. Banigan, E. J., Harris, T. H., Christian, D. A., Hunter, C. A. \& Liu, A. J. 2015 Heterogeneous $\mathrm{CD} 8+\mathrm{T}$ cell migration in the lymph node in the absence of inflammation revealed by quantitative migration analysis. PLoS Comput Biol, 11(2), e1004 058. doi:10.1371/journal.pcbi.1004058.

21. Zaburdaev, V., Denisov, S. \& Klafter, J. 2015 Lévy walks. Rev. Mod. Phys., 87, 483-530. doi:10.1103/RevModPhys.87.483.

22. Loosley, A. J., O’Brien, X. M., Reichner, J. S. \& Tang, J. X. 2015 Describing directional cell migration with a characteristic directionality time. PloS one, 10, e0127425. doi: 10.1371/journal.pone.0127425.

23. Maiuri, P., Rupprecht, J.-F., Wieser, S., Ruprecht, V., Benichoe, O., Carpi, N., Coppey, M., De Beco, S., Gov, N. et al. 2015 Actin flows mediate a universal coupling between cell speed and cell persistence. Cell, 161, 374-386. doi:10.1016/j.cell.2015.01.056.

24. Gorelik, R. \& Gautreau, A. 2015 The arp2/3 inhibitory protein arpin induces cell turning by pausing cell migration. Cytoskeleton (Hoboken, N.J.), 72, 362-371. doi:10.1002/cm.21233.

25. Zenkov, V. S., O'Connor, J., Cockburn, I. A. \& Ganusov, V. V. 2020 A minority of liverlocalized CD8 T cells demonstrate difficult-to-detect attraction to Plasmodium-infected hepatocytes. bioRxiv.

26. Gorelik, R. \& Gautreau, A. 2014 Quantitative and unbiased analysis of directional persistence in cell migration. Nature protocols, 9, 1931-1943. doi:10.1038/nprot.2014.131.

27. Blanchoin, L., Boujemaa-Paterski, R., Sykes, C. \& Plastino, J. 2014 Actin dynamics, architecture, and mechanics in cell motility. Physiological reviews, 94, 235-263. doi: 10.1152/physrev.00018.2013.

28. Genthon, A. 2020 The concept of velocity in the history of brownian motion. The European Physical Journal H, 45(1), 49-105.

29. Purcell, E. M. 1977 Life at low reynolds number. American Journal of Physics, 45(1), 3-11. doi:10.1119/1.10903.

30. Hillen, T., Painter, K. J., Swan, A. C. \& Murtha, A. D. 2017 Moments of von Mises and Fisher distributions and applications. Mathematical biosciences and engineering, 14, 673-694. doi: 10.3934/mbe.2017038.

31. Ulrich, G. 1984 Computer generation of distributions on the m-sphere. Journal of the Royal Statistical Society. Series C (Applied Statistics), 33(2), 158-163. 
bioRxiv preprint doi: https://doi.org/10.1101/2020.12.30.424897; this version posted May 8, 2021. The copyright holder for this preprint (which was not certified by peer review) is the author/funder, who has granted bioRxiv a license to display the preprint in perpetuity. It is made available under aCC-BY-NC-ND 4.0 International license.

32. discontentment, J. M. 2020 Vectors with a certain magnitude in Mathematica. doi: https://mathematica.stackexchange.com/questions/13038/vectorswith-a-certainmagnitude-in-mathematica/13042.

33. Ganusov, V. V., Zenkov, V. S. \& Majumder, B. 2021 Simulating correlated random walks using von Mises-Fisher distribution. https://github.com/vganusov/correlated_random_walk_simulations. b48132e. 


\section{Correlation between speed and turning naturally arises for sparsely sampled cell movements}

Vitaly V. Ganusov, Viktor S. Zenkov, and Barun Majumder

Supplementary Information

A

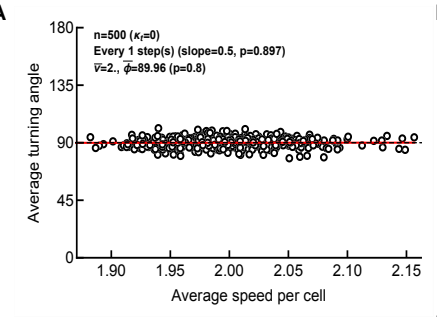

C

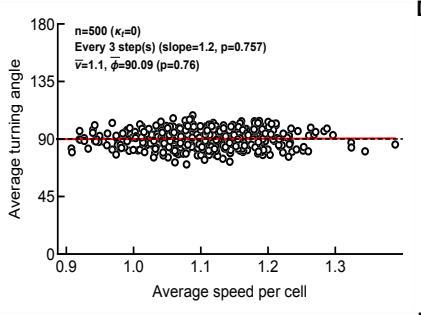

E

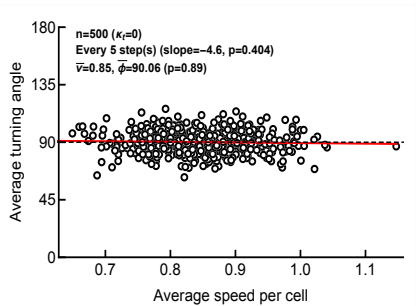

G
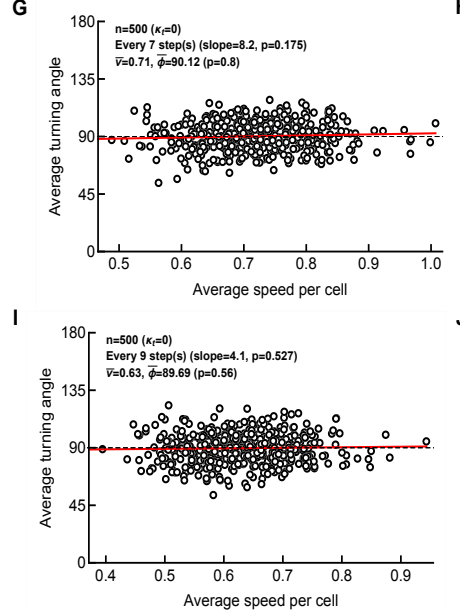

B
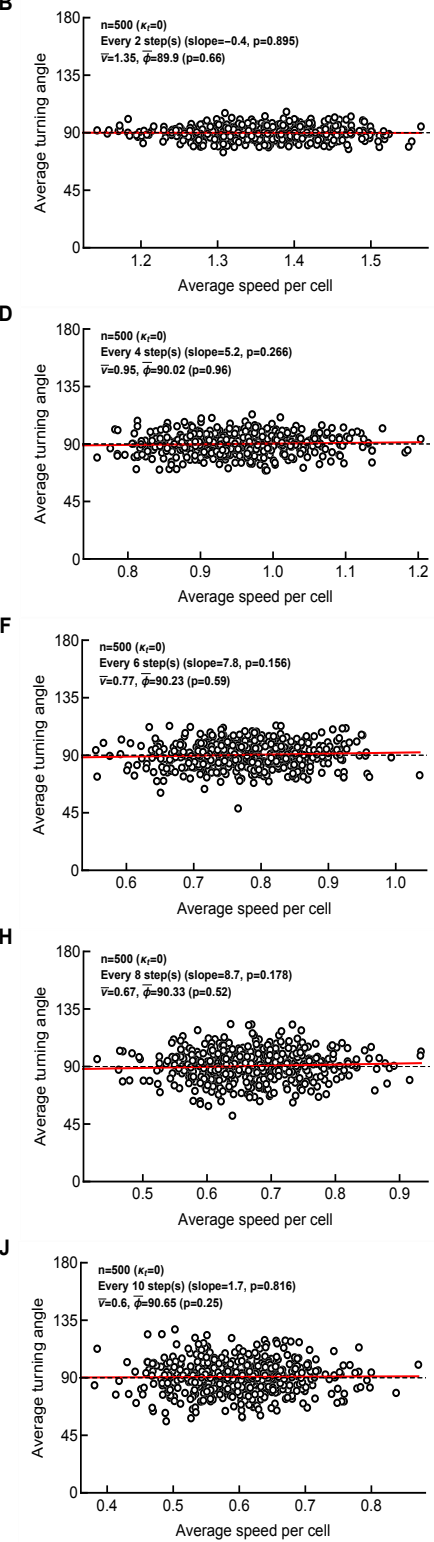

Figure S1: A correlation between average turning angle and average speed does not arise for coarsely sampled data for uncorrelated random (Brownian) walk. Here all cells have the same concentration parameter $\kappa_{t} \rightarrow 0$ (see eqn. (1)). We simulated movements of 500 cells for 100 steps and calculated the average speed and average turning angle per cell when sampling the data at different frequencies, starting with every step (panel A) and finishing with every 10 steps (panel J). Each panel contains information on the average speed for all cells $(\bar{v})$, average turning angle for all cells $(\bar{\phi})$, and the result of linear regression of the average speed per cell and average speed per cell (denoted as "slope") with $p$ value from the t-test. We also provide a test if the average turning angle of cells in the population is different from $90^{\circ}$ (Mann-Whitney test). 
A

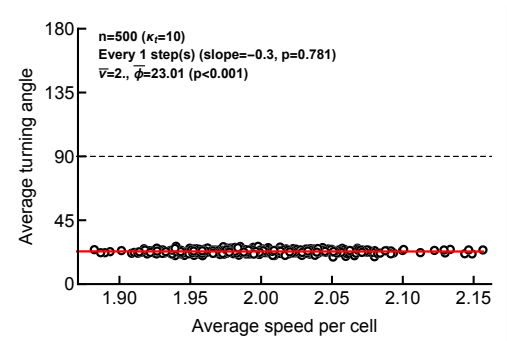

C

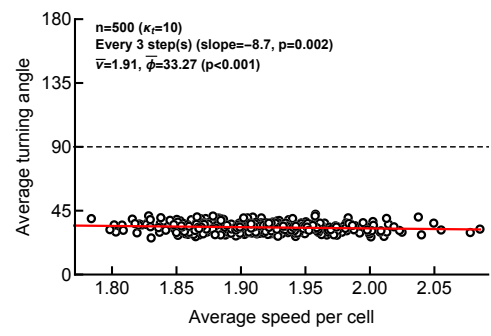

E

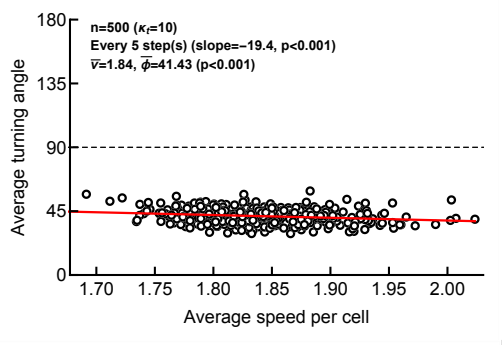

G

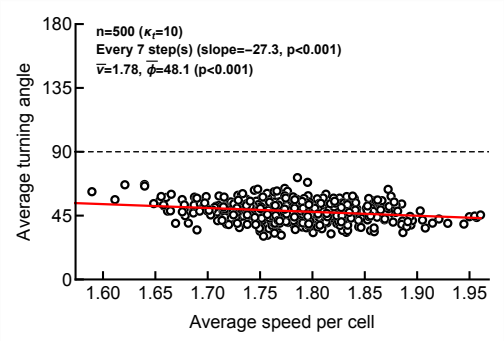

I

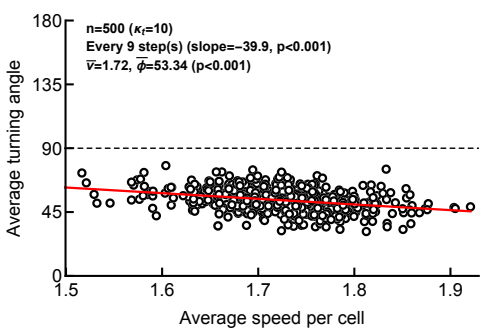

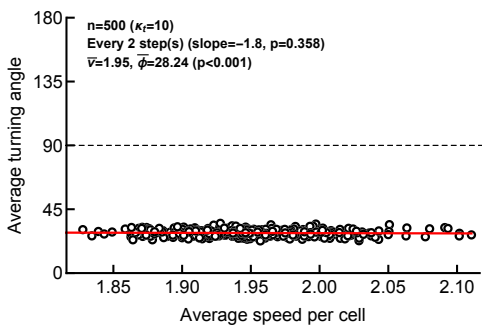
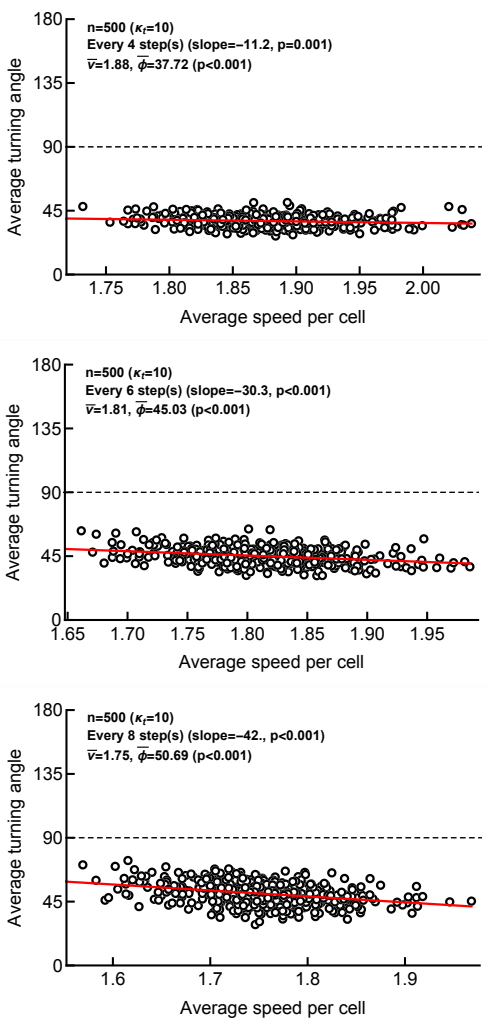

J

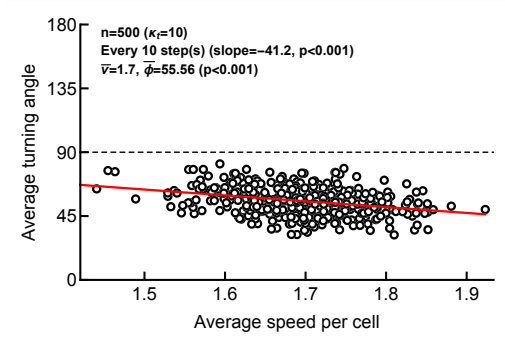

Figure S2: A correlation between average turning angle and average speed appears for coarsely sampled data when cells undergo a correlated random walk. Here all cells have the same concentration parameter $\kappa_{t}=10$ - note different scale on $x$ axis in different panels. See Figure S1 for other details. 
A

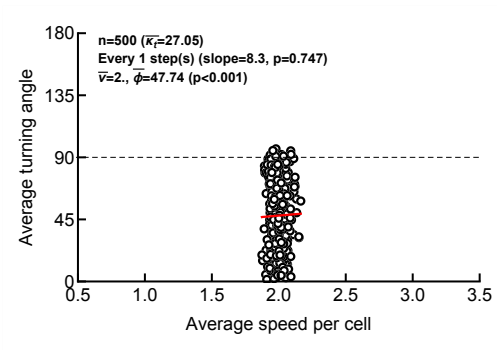

C

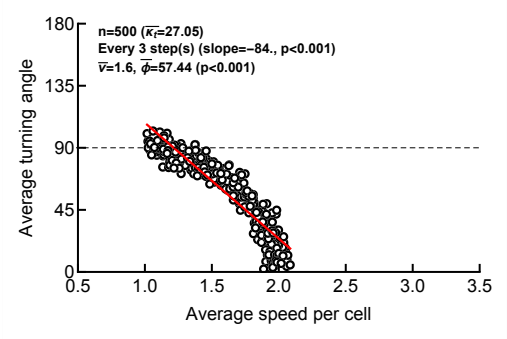

E

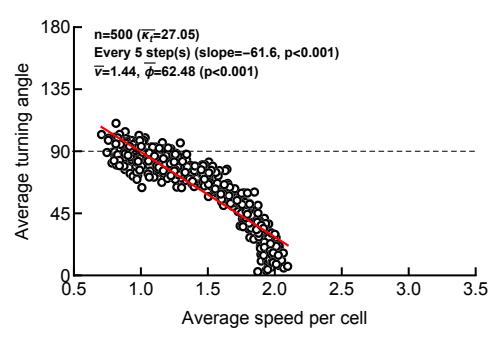

G
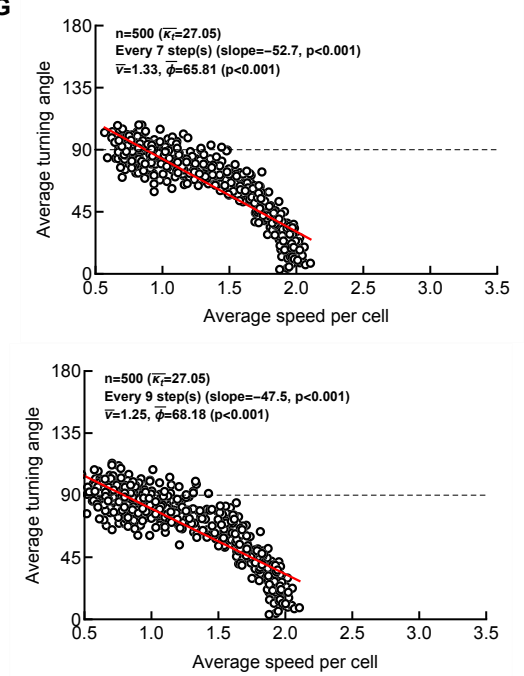
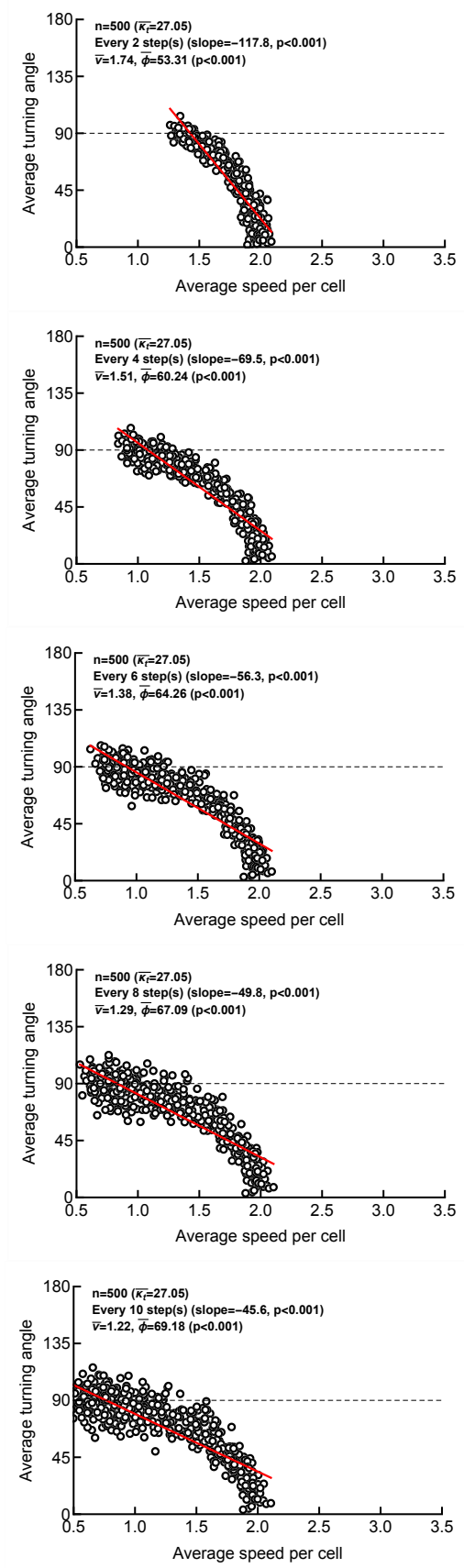

Figure S3: A strong negative correlation between average turning angle and average speed naturally arises for heterogeneous cell populations with coarsely sampled data. Here we assume that every cell in the population has a different $\kappa_{t}$ (from VMF distribution, see eqn. (1)) which was drawn from a lognormal distribution (eqn. (4) with $\mu=1$ and $\sigma=2$ ). Here $\bar{\kappa}_{t}$ is the average concentration parameter in the population. See Figure S1 for other details. 
A

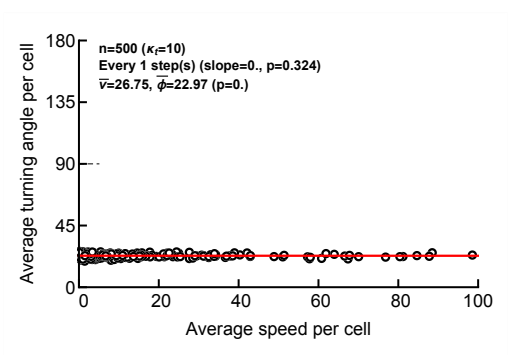

C

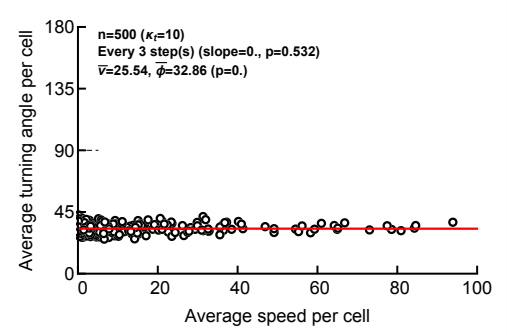

E

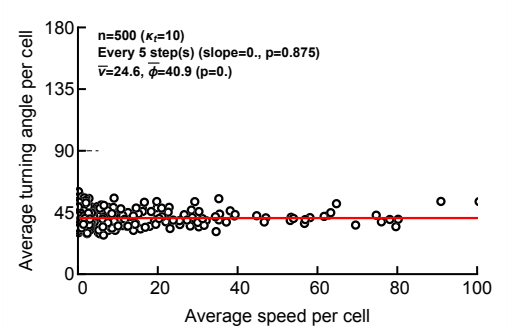

G
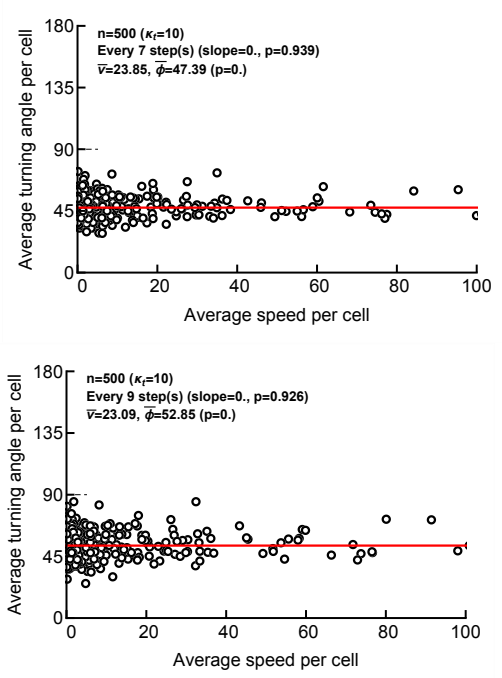
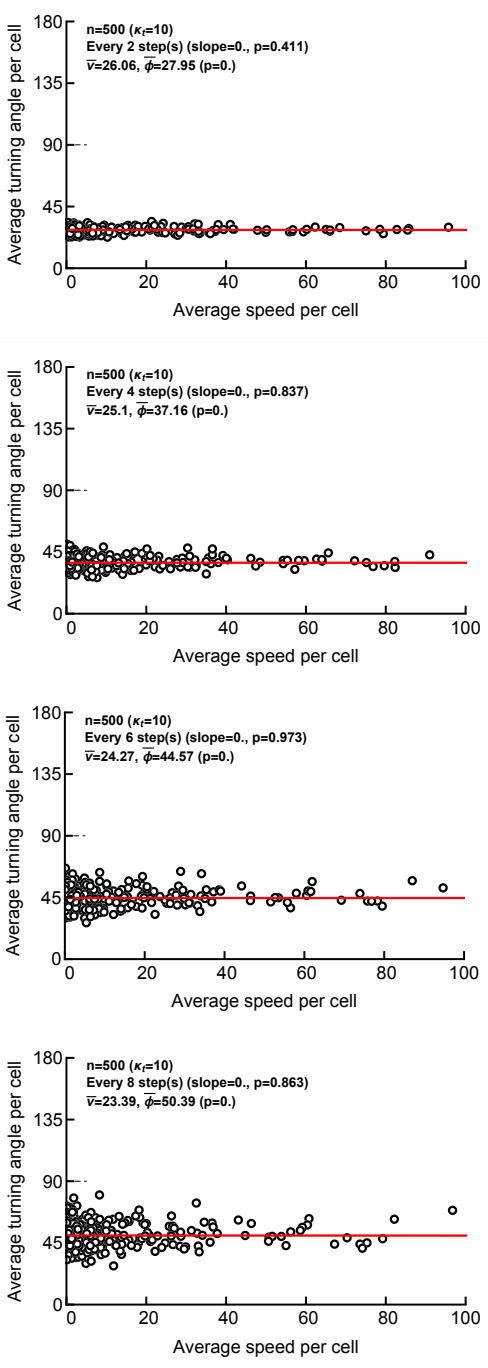

J

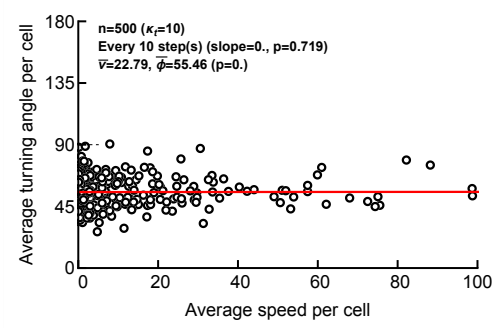

Figure S4: Variability in intrinsic movement speed does not result in a significant correlation between average turning angle and average speed. Here we assume that every cell in the population has a different $\bar{r}$ which was drawn from a lognormal distribution (eqn. (4) with $\mu=1$ and $\sigma=2$ ). Turning angles are defined with $\kappa_{t}=10$ in VMF distribution (eqn. (1)). See Figure S1 for other details. 
A

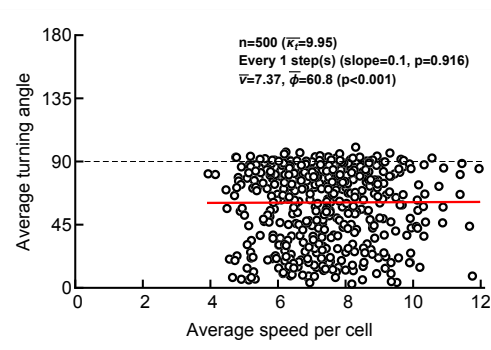

C

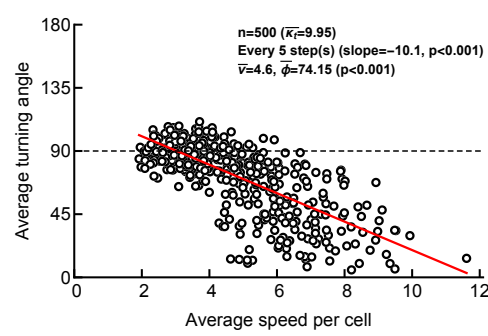

E

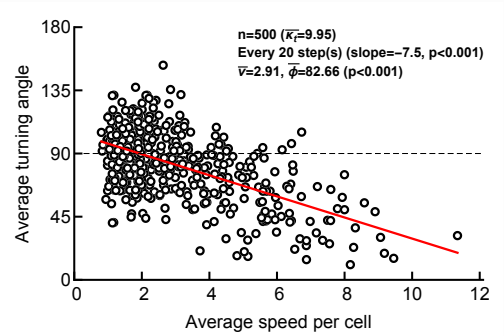

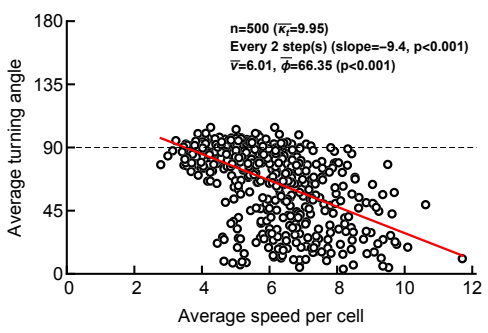

D
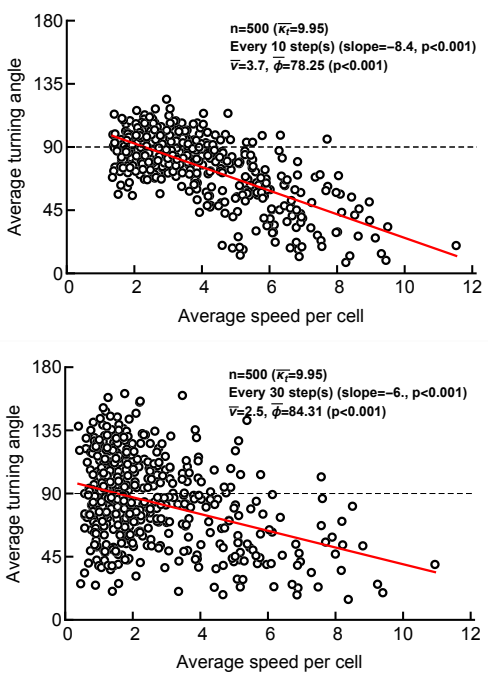

Figure S5: A strong negative correlation between average turning angle and average speed naturally arises when both persistence and cell speeds are variable (but uncorrelated) for different cells for coarsely sampled data. Here we assume that every cell in the population has a different $\kappa_{t}$ which was drawn from a lognormal distribution (eqn. (4) with $\mu=0$ and $\sigma=2$ ), and every cell has a random speed determined by $\bar{r}$ in the Pareto distribution ( $\bar{r}$ was drawn from a lognormal distribution with $\mu=2$ and $\sigma=0.2)$. See Figure S1 for other details. 
bioRxiv preprint doi: https://doi.org/10.1101/2020.12.30.424897; this version posted May 8, 2021. The copyright holder for this preprint (which was not certified by peer review) is the author/funder, who has granted bioRxiv a license to display the preprint in perpetuity. It is made available under aCC-BY-NC-ND 4.0 International license.

A
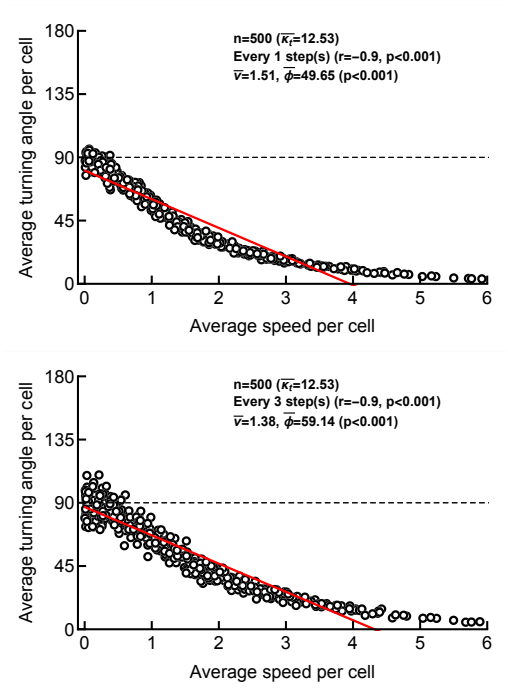

E

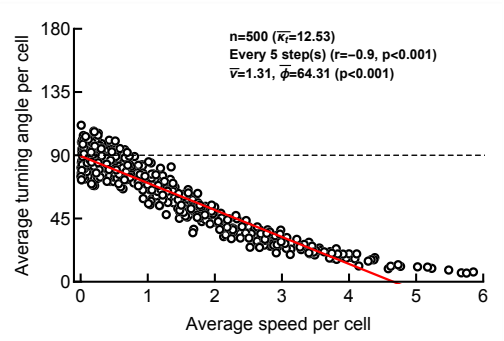

G

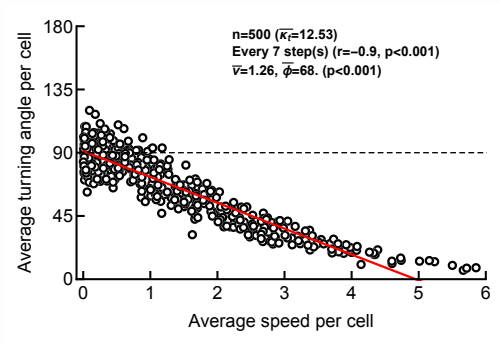

I

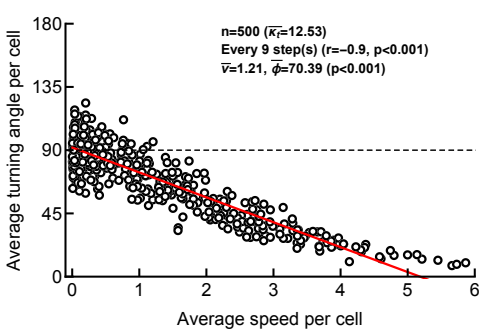

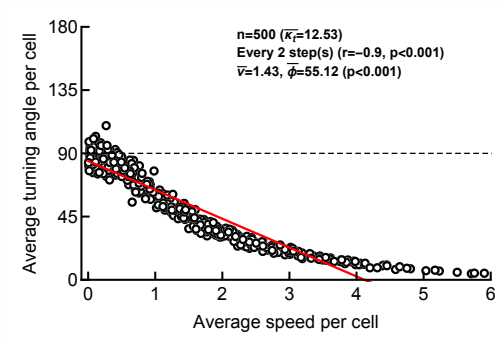
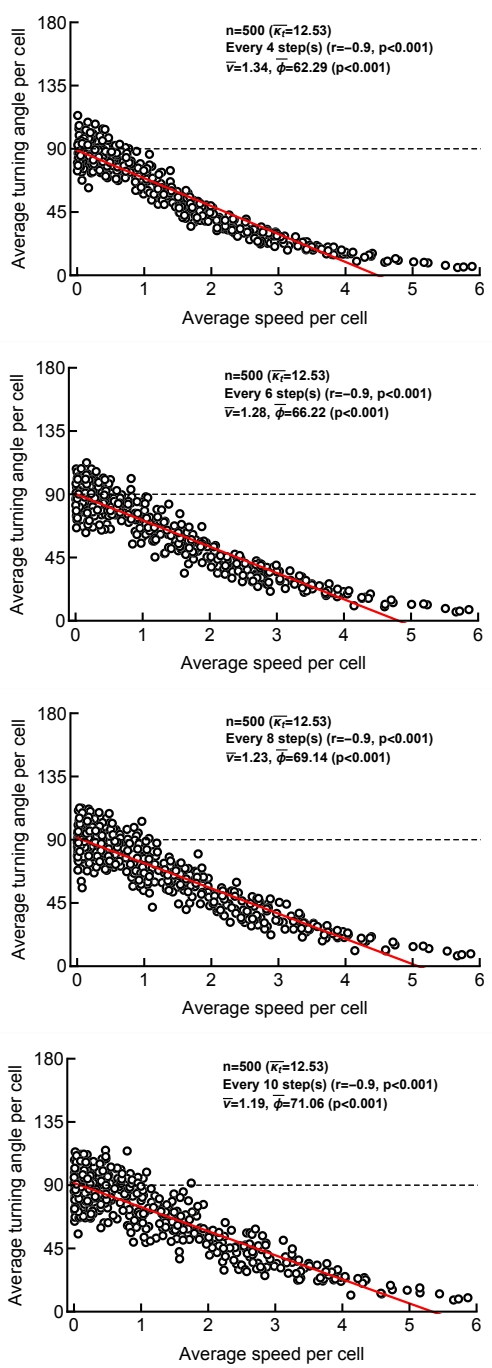

Figure S6: Intrinsic correlation between instantaneous cell speed and turning angles is relativelyt insensitive to sampling frequency. Here we assume that every cell in the population has a different $\kappa_{t}$ which was drawn from a lognormal distribution (eqn. (4) with $\mu=1$ and $\sigma=2$ ), and every cell has a speed determined by $\kappa_{t}$ (i.e., assuming a relationship for each cell as $\bar{r}=\ln \left(1+\kappa_{t}\right)$ for the Pareto distribution (eqn. (3))). See Figure S1 for other details. 
A

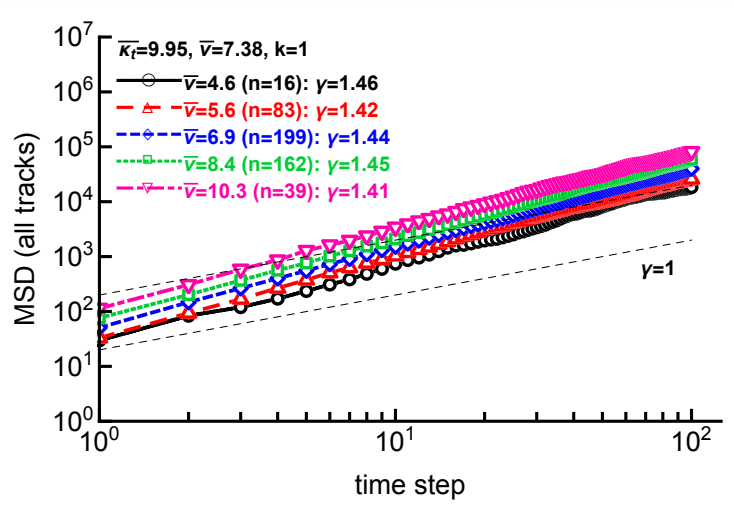

C

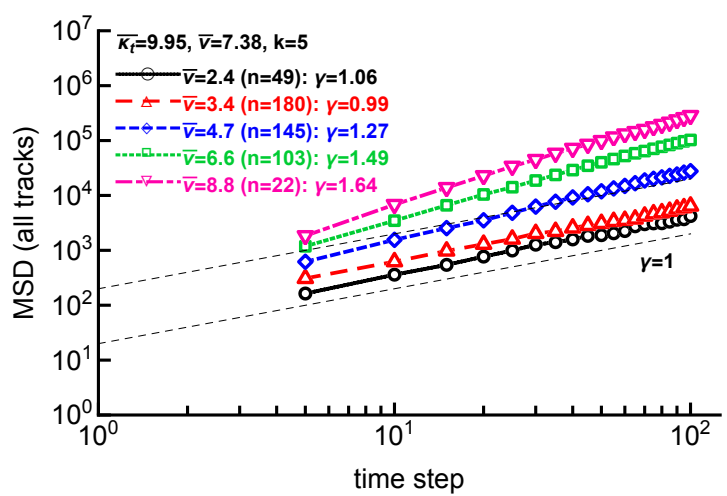

B

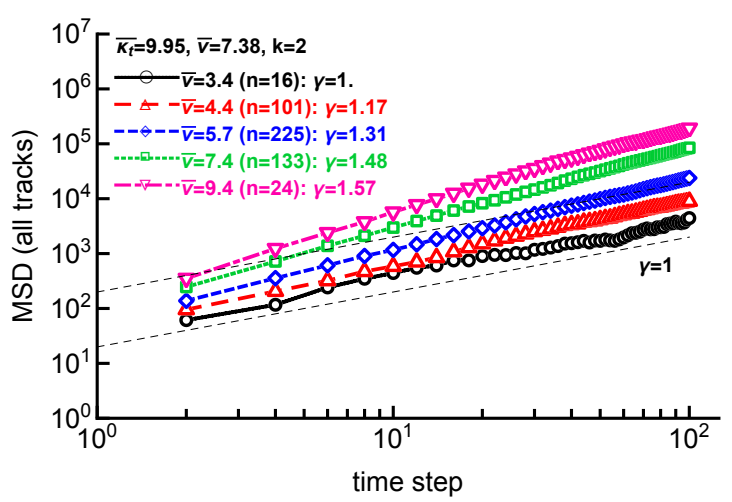

D

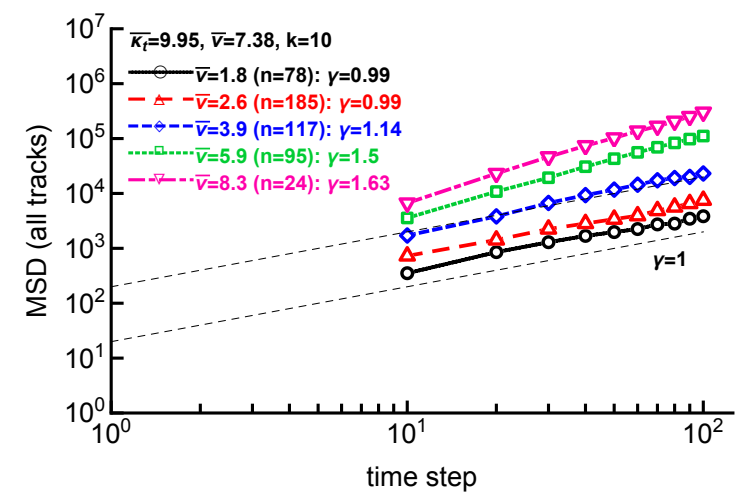

Figure S7: Coarse sampling results in different mean square displacements of cell cohorts with similar inferred average speeds. Here we assume that every cell in the population has a different $\kappa_{t}$ which was drawn from a lognormal distribution (eqn. (4) with $\mu=0$ and $\sigma=2$ ), and every cell has a random speed determined by $\bar{r}$ in the Pareto distribution $(\bar{r}$ was drawn from a lognormal distribution with $\mu=2$ and $\sigma=0.2$ ). This was identical to how simulations were done in Figure S5. We binned the resulting cell trajectories by calculating average speed per track (given specific sampling frequency given by $k$, shown in individual panels) and binned tracks into cohorts with different average speeds. Binning was done by log-transforming the average speeds and then selecting tracks using equally spaced boundaries with 5 bins between minimal and maximal average speeds recorded per sampling simulation. The resulting number of cell trajectories per bin is denoted by $n$ in individual panels, together with the average speed of cells in a bin $(v)$, and the slope $\gamma$ at which log MSD is changing with log time. Simulations were done with 500 cells for 100 timesteps. Thin dashed lines have a slope $\gamma=1$. 
A

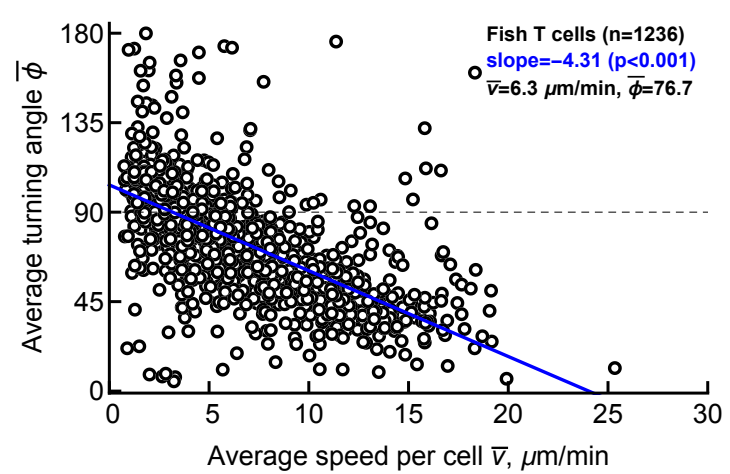

C

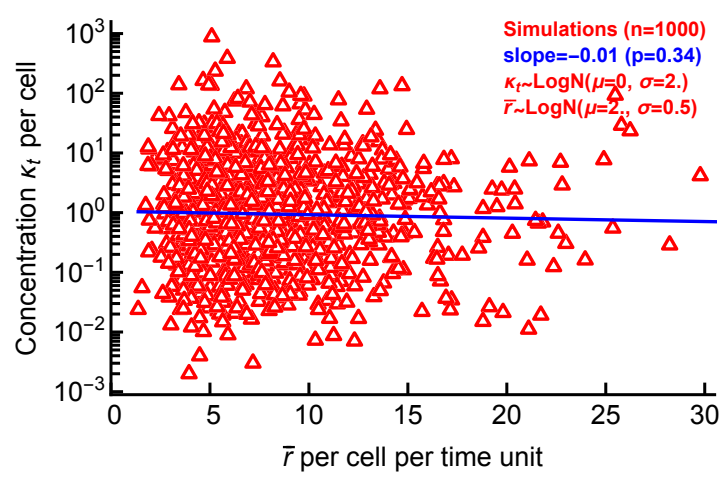

E

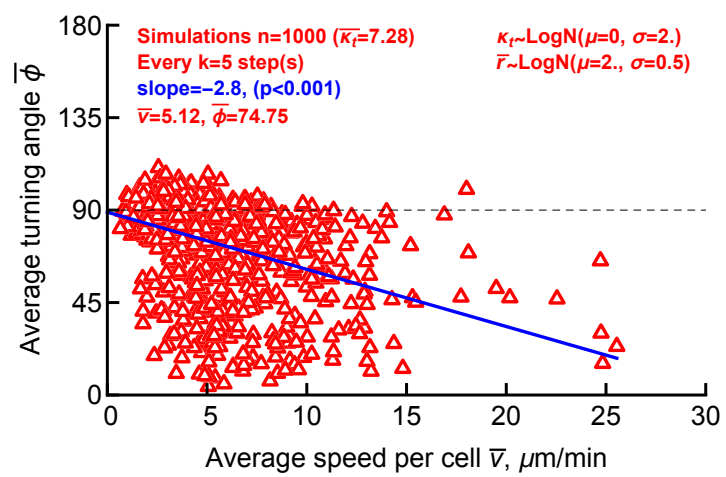

B

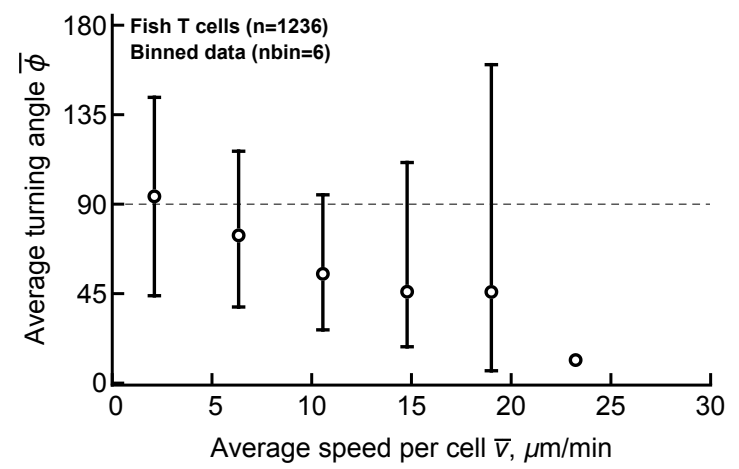

D

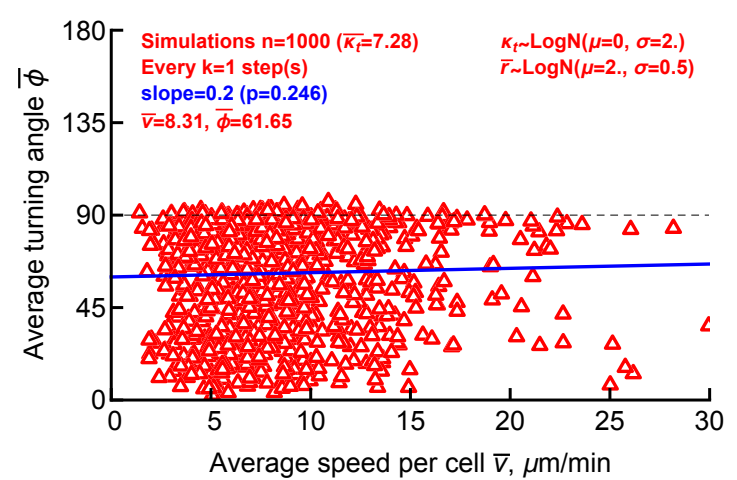

$\mathbf{F}$

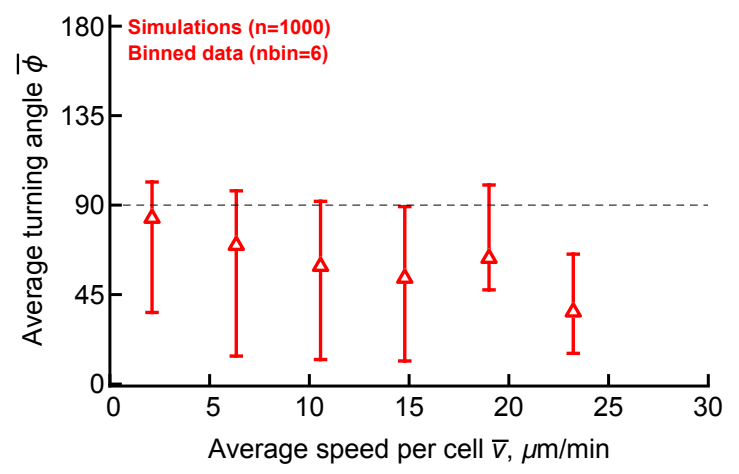

Figure S8: Simulations of cell movement in which cell's speed and turning ability are uncorrelated but are sub-sampled are consistent with experimental data. We cleaned the original data for movement of $\mathrm{T}$ cells in zebrafish [18] by splitting cell tracks that had missing coordinates so that every coordinate measurements occurred in equally spaced intervals (45 sec, see Materials and Methods for more detail). For $n=712$ original cell tracks this resulted in 1236 tracks. For every trajectory we calculated the average speed and average turning angle (A) or binned the data into 6 cohorts with following bin boundaries $(0 ., 4.22,8.45,12.67,16.90,21.12,25.34) \mu \mathrm{m} / \mathrm{min}(\mathrm{B})$. We then performed stochastic simulations of 1000 cells for 100 time units with each cell having a defined persistence ability (defined by $\kappa_{t}$ ) and speed defined by $\bar{r}$, each drawn from a lognormal distribution (eqn. (4)) with parameters $\mu=0, \sigma=2$ for $\kappa_{t}$ and $\mu=2, \sigma=0$. for $\bar{r}$, respectively (C). For every track we then calculated the average speed and average turning angle when the data were sampled every time step $(k=1, \mathrm{D})$ or every $k=5$ steps (E) assuming that sampling in both cases occurs with frequency of $1 \mathrm{~min}$. For the simulations data in $\mathrm{E}$ we also binned the data the same way as for experimental data (F). Confidence intervals in B and $\mathrm{F}$ denote 2.5 and 97.5 percentiles of the data. Other characteristics shown on individual panels are for average speed $\bar{v}$, average turning angle $\bar{\phi}$. $\mathrm{P}$ values for the regression slopes (denoted by solid blue lines) were calculated using linear regression. 
A

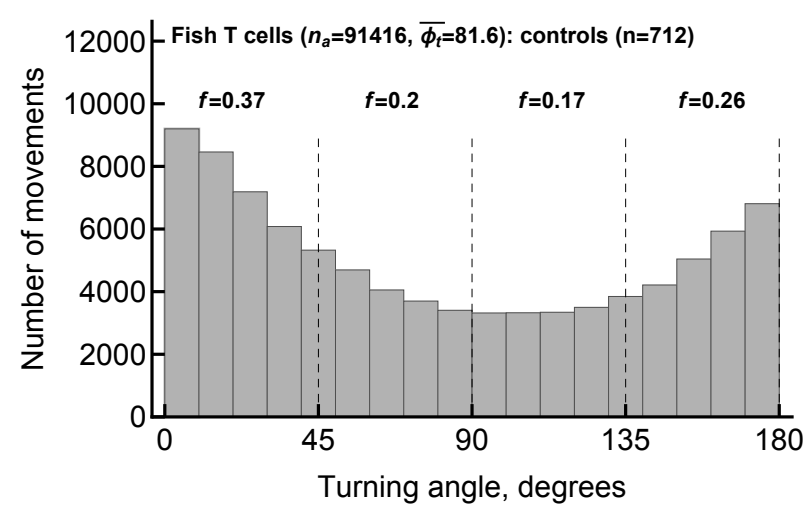

C

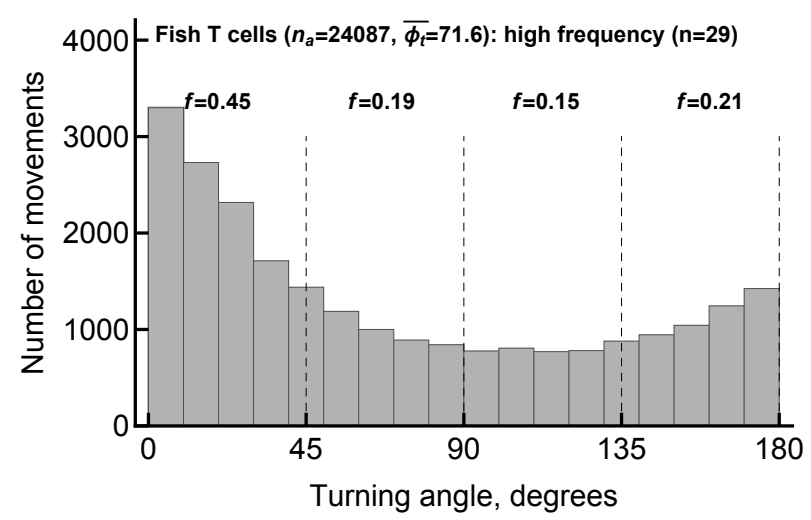

E

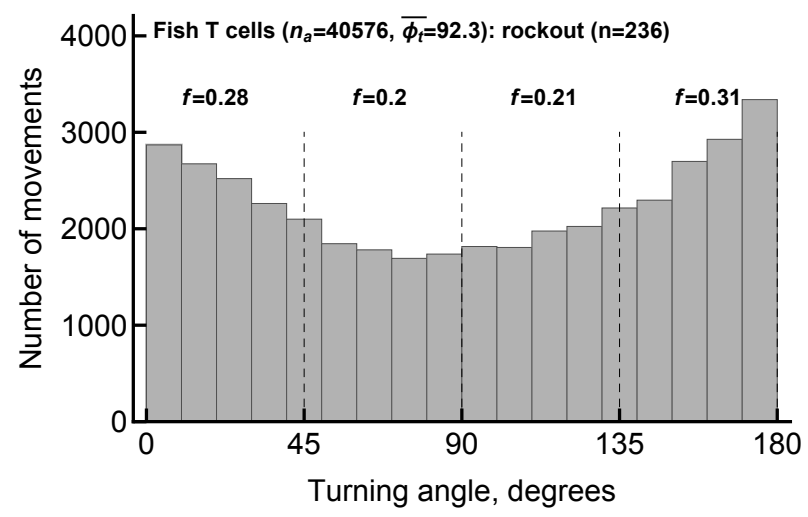

B
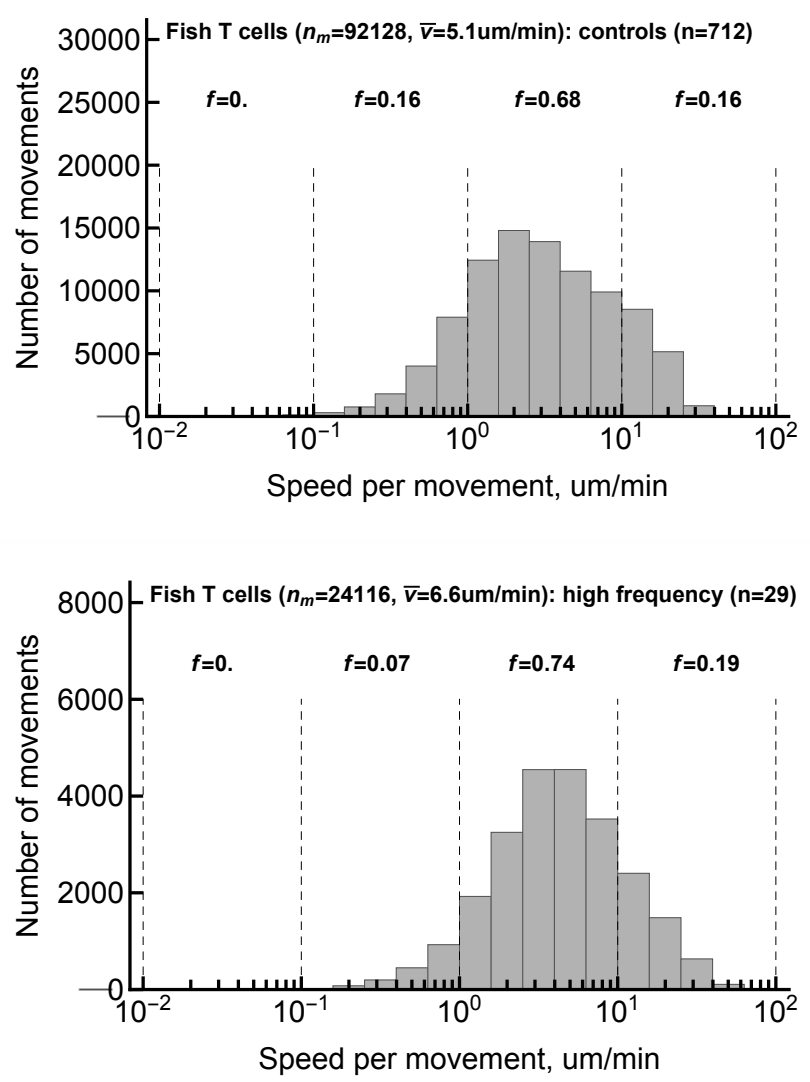

$\mathbf{F}$

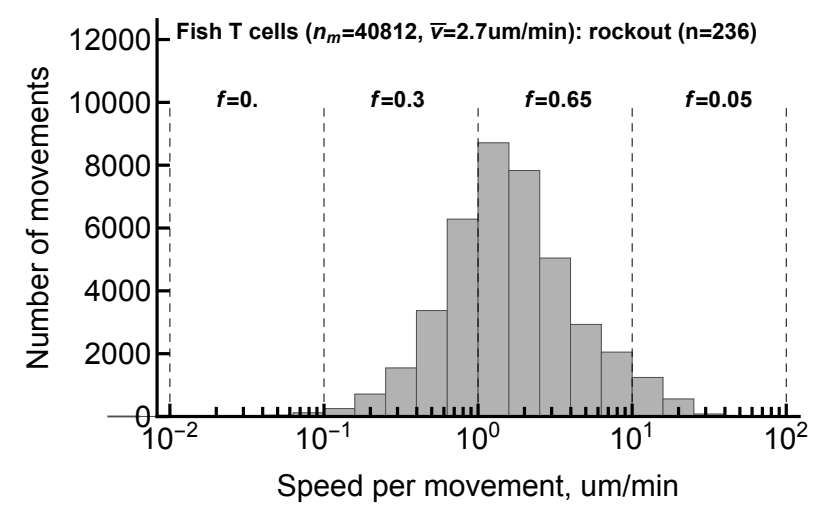

Figure S9: Impact of sampling frequency and cell treatment on movement characteristics of $\mathrm{T}$ cells in zebrafish are consistent with the null model for correlation between speed and turning. We re-analzyed the data on movement of $\mathrm{T}$ cells in zebrafish published recently [18] for control $\mathrm{T}$ cells $(\mathrm{A}-\mathrm{B}, n=712)$, control $\mathrm{T}$ cells with measurements done at high frequency (every 12 sec, C-D, $n=29$ ), or $\mathrm{T}$ cells treated with Rockout, Rho kinase inhibitor III (E-F, $n=236$ ). For each cell movement (or a pair of cell movements) we calculating turning angles $(\mathrm{A}, \mathrm{C}, \mathrm{E})$ or speeds $(\mathrm{B}, \mathrm{D}, \mathrm{F})$; the average turning angle $\left(\bar{\phi}_{t}\right)$ and average speed $(\bar{v})$ per movement along with the total number of angles $\left(n_{a}\right)$ and movements $\left.n_{m}\right)$ are shown on individual panels. In all panels we show the proportions of measurements that fall into specific bin (e.g., in panel A, $37 \%$ of movements have turning angles between 0 and $\left.45^{\circ}\right)$. In panels $n_{a}$ is the number of angles, and $n_{m}$ is the number of movements. 